\title{
Rare and Endangered Plant Leaf Identification Method Based on Transfer Learning and Knowledge Distillation
}

\author{
Lin Wu, School of Information Science and Engineering, Hebei North University, China \\ Jingjing Yang, School of Information Science and Engineering, Hebei North University, China \\ (iD https://orcid.org/0000-0003-2751-2930 \\ Zhihao Gu, School of Information Science and Engineering, Hebei North University, China \\ Jiaqian Guo, School of Information Science and Engineering, Hebei North University, China \\ Xiao Zhang, School of Information Science and Engineering, Hebei North University, China \\ (iD) https://orcid.org/0000-0002-8592-8160
}

\begin{abstract}
Considering the limited sample size of rare and endangered plant leaves and the issue that leaf identification is mainly conducted using mobile smart devices and other technology with low computing power, this paper proposes a rare and endangered plant leaf identification method based on transfer learning and knowledge distillation. Following the expansion of data sets containing rare and endangered plant leaves, the last fully connected layer was replaced with trained Alexnet, VGG16, GoogLeNet, and ResNet models to conduct transfer learning, and realize a relatively high success rate in identifying images of these species. Then, knowledge distillation was utilized to transfer Alexnet, VGG16, GoogLeNet, and ResNet models into a lightweight model. The experiment results indicate that, compared with other methods, the lightweight rare and endangered plant identification model trained with the methods described in this paper was not only more accurate but also less complex than its alternatives.
\end{abstract}

\section{KEYWORDS}

Convolutional Neural Network (CNN), Image Identification, Knowledge Distillation, Leaf Identification, Lightweight Network Model, Low Computing Power, Rare and Endangered Species, Transfer Learning

\section{INTRODUCTION}

Plants are an important part of ecological systems and biodiversity. Protecting plant diversity can benefit the sustainable development of human society (Narzullaev, 2021). Due to the immoderate utilization of wild plant resources, many wild plants are being deracinated rapidly, which severely hurts the balance of the ecological environment on Earth (Fan et al., 2018; Xu et al., 2017). In the meantime, damage to the ecological system is also reducing living space for animals, further accelerating species extinction (Roberts, Hassan, Elamer, \& Nandy, 2021). The extinction of each 
plant species would cause the deracination of 10-30 neighboring species, resulting in a serious impact on the ecological system (Ceballos, Ehrlich, \& Raven, 2020). As the number of endangered plants is declining for different reasons, any species extinction could cause damage to the entire ecological system, and the lives and development of humans are threatened. It is necessary to take protective actions as soon as possible to avoid the risk of these extinctions ( $\mathrm{Li}$ et al., 2018).

Effective protection for rare and endangered plants includes in situ conservation, namely, to build nature reserves in the primitive environment and reduce artificial interference and damage to realize the goal of protecting these species (Mounce, Smith, \& Brockington, 2017). Due to the wide required scope of knowledge, relevant personnel are facing difficulties in identifying endangered plants. This escalates the degree of difficulty of endangered plant protection. Significant efforts and follow-up are urgently needed (Liu, 2021). It has become important to mitigate germplasm resource loss caused by difficulties for personnel in endangered plant identification by adopting automatic identification based on computer, artificial intelligence, and other similar technologies.

Blooms, leaves, and fruit could all be used as evidence for endangered plant identification. Among these parts, leaves have become the major method for plant identification, as they are stable in structure, easy to be collected, and have long life cycles. However, artificial identification of leaves requires a lot of work and is inefficient. It is a subjective method and requires a wider scope of knowledge than other identification practices. So far, there has already been some research on automatic leaf identification methods. These methods mainly identify leaves by their superficial characteristics, such as color, shape, and texture (Saleem, Akhtar, Ahmed, \& Qureshi, 2019; Singh, Nagahma, Yadav, \& Yadav, 2018; Yang, 2021). Most plant leaves are green, which means there is no obvious difference between plants. In contrast, shapes do have obvious differences, even on the same plant. Textures are too difficult to describe and to use to yield conclusions. Therefore, these adopted superficial characteristics lack universality. Models calculated based on such characteristics are also incapable of being used for generalization. It has become common for recent research to automatically extract deep features of leaves through deep learning (Lee, Chan, Mayo, \& Remagnino, 2017; Lin, Ding, Tu, Huang, \& Liu, 2019). Due to the small sample size of plants, especially endangered plants, in real applications, the direct adoption of the deep learning model may lead to overfitting. Transfer learning is thus used in the plant leaf identification model (Gogul \& Kumar, 2017; Kaya et al., 2019). However, the large number of parameters created for these models after transfer learning requires more computing resources. In outdoor environments and other scenes without network and server support, it would be difficult to apply them to smart mobile terminals and other technologies with low computing power. Moreover, no research specially focuses on rare and endangered plant leaf identification.

In response to these issues, this paper proposes an endangered plant leaf identification method based on transfer learning and knowledge distillation, which guarantees high accuracy and requires fewer convolutional neural network (CNN) parameters. The lightweight endangered plant leaf identification model is proposed for application to more settings. The major contributions of this paper are as follows:

1. This paper trained a CNN with an endangered plant leaf identification data set to enable the learning of deep features of leaf images. This solved the issues of the low accuracy of artificial leaf identification using superficial characteristics and the absence of having a specialized endangered plant leaf identification model.

2. Transfer learning is used as a solution for overfitting in CNNs caused by the limited sample size of endangered plant leaves. In the meantime, the transfer model enables the generalization of deep features, which is more accurate than using the directly trained CNN.

3. The lightweight endangered plant leaf identification model after knowledge distillation performs similarly to the complex transfer learning network identification model, yet its parameters are only $5.64 \%-19.00 \%$ of the complex object detection network. It promotes the performance of 
the network model and extends the application scenarios of endangered plant leaf identification models.

The rest of the paper is arranged as follows. Section 2 includes the literature review. Sections 3 and 4 describe the methodology and experiments. Section 5 serves as the conclusion.

\section{LITERATURE REVIEW}

Plant leaf identification methods mainly include methods based on feature engineering and leaf identification network models based on deep learning. Regarding the former, (Zhang, Zeng, \& Zhang, 2019) extracted shape and color features of plant leaves and applied classification methods, including random forest, LDA, logistic regression, and sparse representation algorithms. Their results proved that the random forest algorithm is the most accurate. (Islama, Yousuf, \& Billah, 2019) proposed a kind of leaf identification method that uses a histogram of oriented gradients (HOG) and local binary patterns (LBP) to extract the feature named Flavia Leaf Data Set to train the support vector machine (SVM) model, which exhibited good performance. Other work (Chaki, Dey, Moraru, \& Shi, 2019; Yang, 2021) also used shape, color, and texture to extract leaf features for classification.

(Ibrahim, Sabri, \& Abu Mangshor, 2018) built a data set with about ten kinds of herbaceous plant. Three texture features-LBP, SURF, and HOG-were extracted and entered the SVM for leaf identification. LBP and HOG were the most accurate. (Saleem et al., 2019) also evaluated visual features of different leaves and the performance of different classification methods. The results indicated that the k-nearest neighbor had the best performance. Leaf recognition methods based on feature engineering are also based on solidity, circularity, convexity and eccentricity, etc.(Kadir, 2014; Kadir, Nugroho, Susanto, \& Santosa, 2011, 2013; Mata-Montero \& Carranza-Rojas, 2015). The identification performance of leaf identification methods based on feature engineering relies to a great extent on the reasonableness of artificially selected features, which hides blindness. Therefore, leaf identification network models based on deep learning have become popular in research. But a new feature set for shape-only leaf recognition was proposed. Many publicly available leaf data sets were evaluated, and classification accuracy of more than $90 \%$ was achieved on most data sets (Hewitt \& Mahmoud, 2018).

(Pawara, Okafor, Surinta, Schomaker, \& Wiering, 2017) used a CNN-based method to classify a plant image data set. They found that CNN performed better than artificial extraction methods. Due to the difficulty in collecting more plant leaf samples in real applications, other scholars (Beikmohammadi \& Faez, 2018; Kaya et al., 2019) proposed using a transfer learning method that learns small-sample plant leaf data sets with pre-trained models. This method could realize good performance in plant leaf identification. Some work (Parvatikar \& Parasar, 2021) used a CNN model to extract leaf vein features of plant leaves and proved that increasing $\mathrm{CNN}$ depth could effectively promote the accuracy of plant leaf classification. Though the leaf identification network model based on deep learning enables automatic leaf feature extraction and produces results that are generalizable, this model has many parameters, which creates serious demands on computing resources.

\section{METHODOLOGY}

We utilize a rare and endangered plant leaf data set $X=\left\{\left(x_{1}, y_{1}\right),\left(x_{2}, y_{2}\right), \cdots \cdots\left(x_{n}, y_{n}\right)\right\}$, where $n$ represents the size of the data set, $x_{i} \in X$ represents the images of the plant leaves, and $y_{i} \in X$ is a tag of $x_{i}$, namely, the species of $x_{i}$. Identification model $M$ has to satisfy the following: 


$$
M(x)=\left\{\begin{array}{lc}
y_{i} & x=x_{i} \in X \\
\text { null } & x \notin X
\end{array}\right\}
$$

Namely, the identification model $M$ could precisely identify the tag to which $x_{i}$ corresponds.

In this paper, we propose three kinds of $\mathrm{CNN}$-based rare and endangered plant leaf identification models, $M$, as shown in Figure 1. Figures 1a-1d display the pre-trained models that use Alexnet (Krizhevsky, Sutskever, \& Hinton, 2017), VGG16 (Qassim, Verma, \& Feinzimer, 2018), ResNet (He, Zhang, Ren, \& Sun, 2016), and GoogLeNet (Szegedy, Vanhoucke, Ioffe, Shlens, \& Wojna, 2016), respectively, for transfer learning. Figure 1(e) is the lightweight endangered plant leaf identification model after knowledge distillation. We used the pre-trained models in Figures 1a-1d to finish knowledge transfer training from abundant images to endangered plant leaves. Then, we used the model in Figure 1(e) to conduct knowledge distillation on the models in Figures 1a-1d, and to produce output using the Alexnet, VGG16, GoogLeNet, and ResNet knowledge distillation models. Next, we discuss two aspects of the CNN identification model based on transfer learning and the lightweight identification model based on knowledge distillation.

\section{Rare and Endangered Plant Leaf Identification Model Based on Transfer Learning}

Alexnet and VGG16 are classic deep CNN structures that achieve high accuracy in identifying largescale image data sets. Both models are constituted by several convolutional layers (Conv 2d), pooling layers (Max Pool 2d), fully connected layers (Dense), flatten layers (Flatten), and Softmax. As shown in Figures 1(a) and 1(b), the number of plant leaf images is represented as n, with a resolution of $150 \times 150$, and there are three channels. After the normalization process, the data were entered into the convolutional layer, represented as:

$$
h^{m(l)}(x)=\operatorname{ReLU}\left(b^{m(l)}+\sum_{n} W^{m n(l)} * h^{n(l-1)}\right)
$$

where, $h$ refers to the fitting function output of the convolutional layer; $x$ refers to the image of the plant leaf; $b$ is the regularizer; $W$ is the parameters of the network model; $m, n$ refers to the serial number of the fitting function for different layers; $l$ refers to the number of layers of the neural network; and * is the convolutional sign. Through the convolutional layer, local and global features of plant leaf image $x$ can be automatically extracted by sliding the window. ReLU is the activation function, and its function is to make some neuron reach zero to spare the network, reduce interdependence connection between parameters, and thus mitigate overfitting.

The output of the convolutional layer is entered into the pooling layer, represented as:

$h_{(i, j)}^{m(l)}=\max _{\forall(p, q) \in U_{i, j}}\left\{h_{(p, q)}^{m(l)}\right\}$

where $U_{i, j}$ refers to the regions indexed as $i, j$ and $p, q$ is the actual index. The max pooling method was adopted to reduce the dimensions of deep features of plant leaf image $x$ extracted from the convolutional layer, thus accelerating the convergence rate of the model.

After several convolutional layers and pooling layers, the response value matrix for plant image $x$ was entered into the fully connected layer (Dense) and the flatten layer (Flatten). The fully connected layer conducted dimension reduction on the deep feature vector, and mapped the distributed characteristic learned by the models into the label space of the plant leaf image data set. The Flatten 


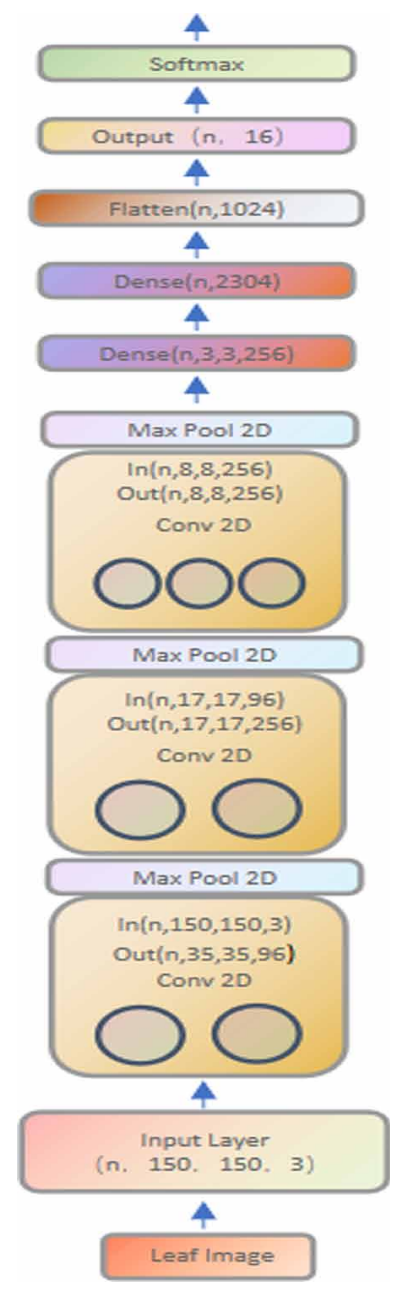

layer was used to transfer multi-dimensional deep features into one dimension so that the Softmax function could be used to anticipate the multi-classifications.

To improve accuracy in rare and endangered plant leaf identification and achieve a faster convergence rate, we needed to use transfer learning to finish knowledge transfer training from abundant images to rare and endangered plant leaves. Therefore, trained Alexnet and VGG16 models were used to learn specific features of endangered plant leaves with extracted generalized visual features. The process is shown in Figures 1a-1d, where the output layer is the fully connected layer. The parameters of all the convolutional layers (Conv 2d) and pooling layers (Max Pool 2d) before the output layer were frozen, as the image classification identified by the last layer of the output layer was inconsistent with the data set of the rare and endangered plant leaves. The last layer of the output layer was thus replaced and configured as the same classification as the rare and endangered plant leaf; only the fully connected layer (Dense), the flatten layer (Flatten), and the Softmax layer were trained.

Rare and endangered plant leaf identification is a multi-classification task, so network model loss was defined as: 


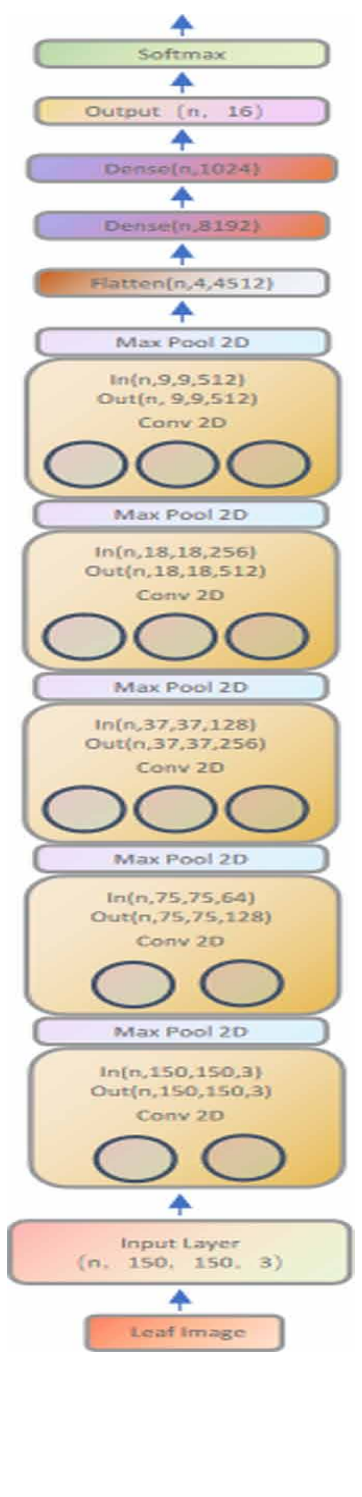

where $y_{i}$ is the real classification tag of $x_{i}$, and $h_{i}$ is the output of the last layer of the output layer of the network model.

\section{Lightweight Rare and Endangered Plant Leaf Identification Model Based on Knowledge Distillation}

Knowledge distillation refers to trainings that use high-accuracy complex teacher network models to train lightweight student network models. It allows student models to maintain a relatively high identification success rate with a small number of parameters. The lightweight student network model could be deployed to mobile smart terminals and embedded devices, which is convenient for plant leaf identification in the wild. As shown in Figure 2, the first step was to use wild rare and endangered plant leaf images and corresponding tags to train the teacher network through transfer learning, as 
Figure 1c. Structures of the five rare and endangered plant leaf identification models

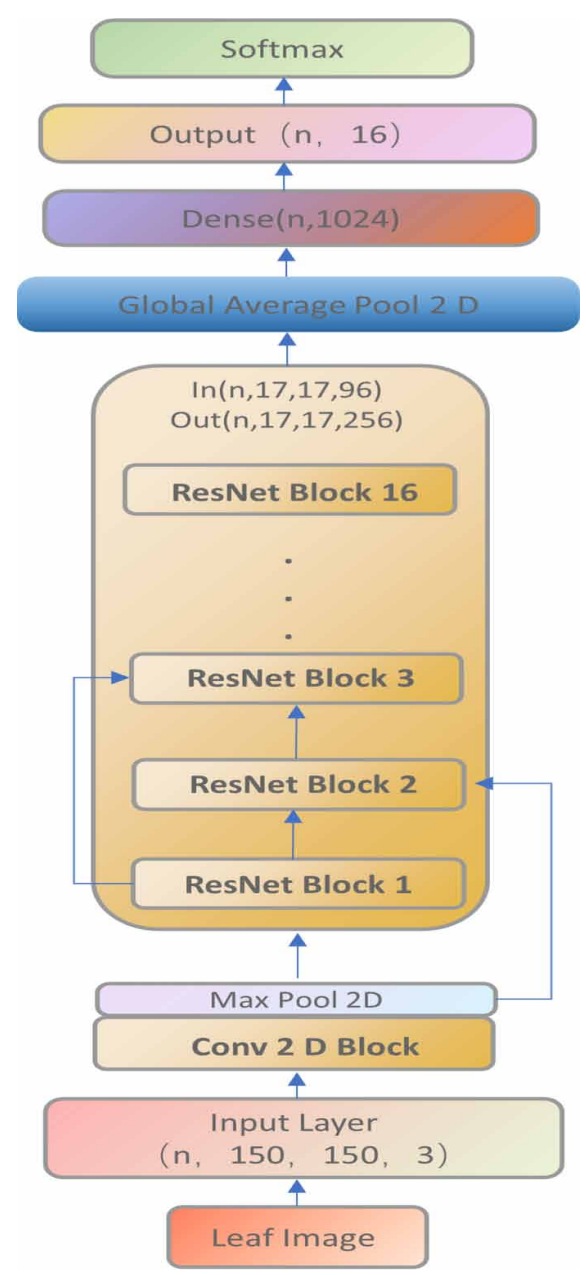

discussed above. Then, the teacher network was used to train the student network model. The critical part was introducing the soft label as part of the loss function (Hinton, Vinyals, \& Dean, 2015), which could better train the student model. To introduce the soft label, a temperature variable $T$ was introduced for softening. The soft label was adopted as the input for the Softmax layer, as follows:

$q_{i}=\frac{\exp \left(h_{i} / T\right)}{\sum_{j} \exp \left(h_{j} / T\right)}$

where $q_{i}$ indicates the soft-label output of a type $i$ rare and endangered plant after the Softmax layer.

During the process of student network model training, soft-label and real plant leaf tags trained the student network at the same time. Knowledge of the teacher network model had already been transferred to the student network model. Therefore, the loss function for the process of student network model training is: 
Figure 1d. Structures of the five rare and endangered plant leaf identification models

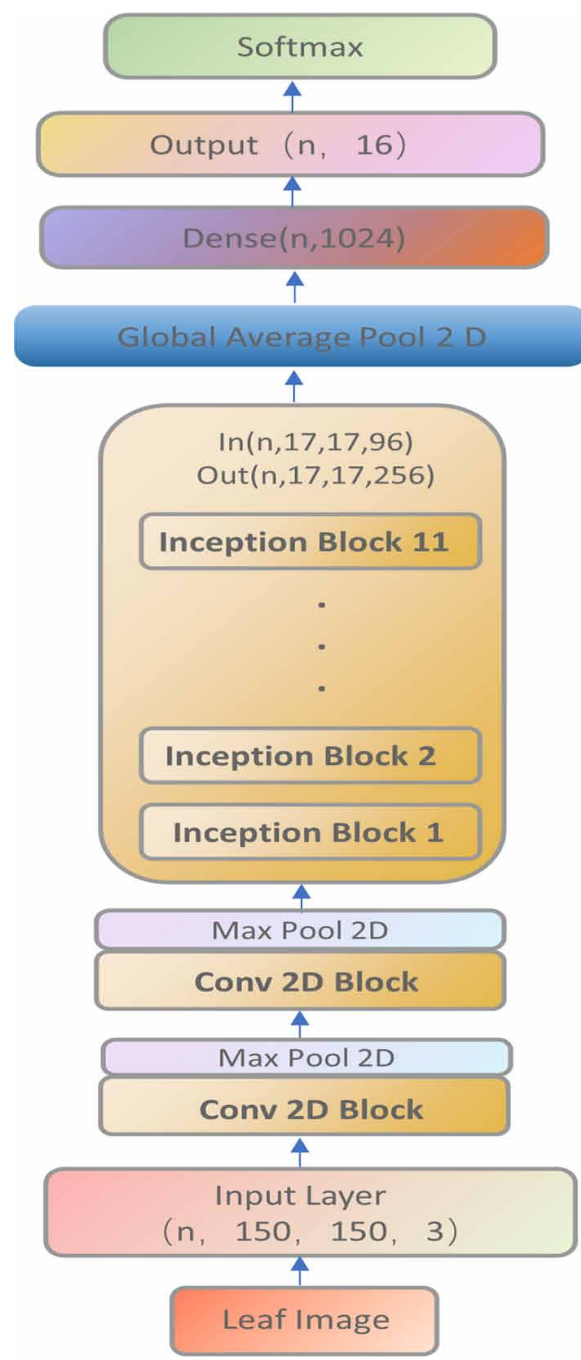

$L_{2}=\chi_{1} L_{\text {soft }}+\chi_{2} L_{\text {hard }}$

where $\chi_{1}$ and $\chi_{2}$ are hyper parameters; $L_{\text {soft }}$ is the multi-classification cross-entropy loss function for student network model training by the soft label; and $L_{\text {hard }}$ is the cross-entropy loss function for student network training using the real rare endangered plant leaf tags.

The structure of the student network model trained in this paper is shown in Figure 1(c). The depths of the network models were all smaller than those for Alexnet and VGG16. To use fewer parameters, the adoption of knowledge distillation technology resulted in an accuracy rate for endangered plant leaf identification for the student network model that was similar to Alexnet and VGG 16. 


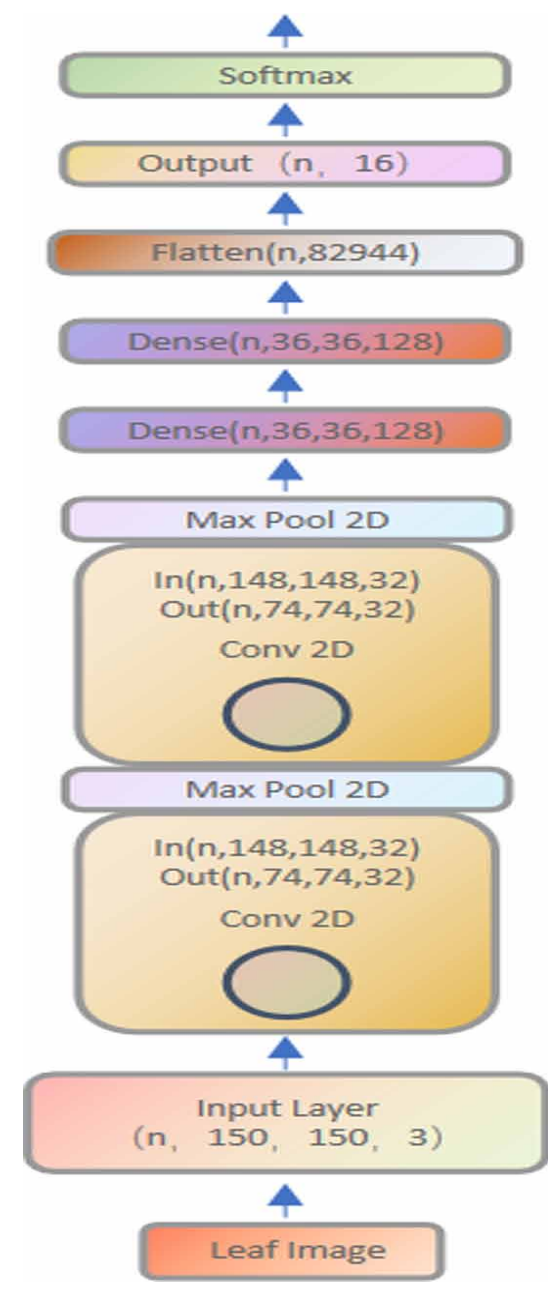

\section{EXPERIMENT}

\section{Dataset}

The rare and endangered plant leaf image data set was downloaded from the Information System of Chinese Rare and Endangered Plants and (Kumar et al., 2012). It includes the following 16 kinds of rare and endangered plants: Bretschneidera sinensis, Aquilaria sinensis, Cinnamomum pedunculatum, Tetracentron sinense, Euryodendron excelsum, Davidia involucrata, Cercidiphyllum japonicum, Liriodendron chinense, Chionanthus retusus, Sassafras albidum, Magnolia virginiana, Zelkova serrata, Metasequoia glyptostroboides, Pinus koraiensis, Chionanthus virginicu, and Magnolia dandyi. The dataset includes 2,620 images with resolutions of $150 \times 150$. Using the Sobel operator to segment the images leaves only the leaves. To mitigate overfitting, as shown in Figure 3, the ImageDataGenerator function in the keras library was used to conduct pretreatment, including normalization, random resizing, rotation, cut, horizontal flip, and other processes. After 1,000 iterations, an expanded leaf image data set of 2,620,000 images was obtained. The data set was then randomly divided into a test set, a verification set, and a test set, with a ratio of 60:20:20. 


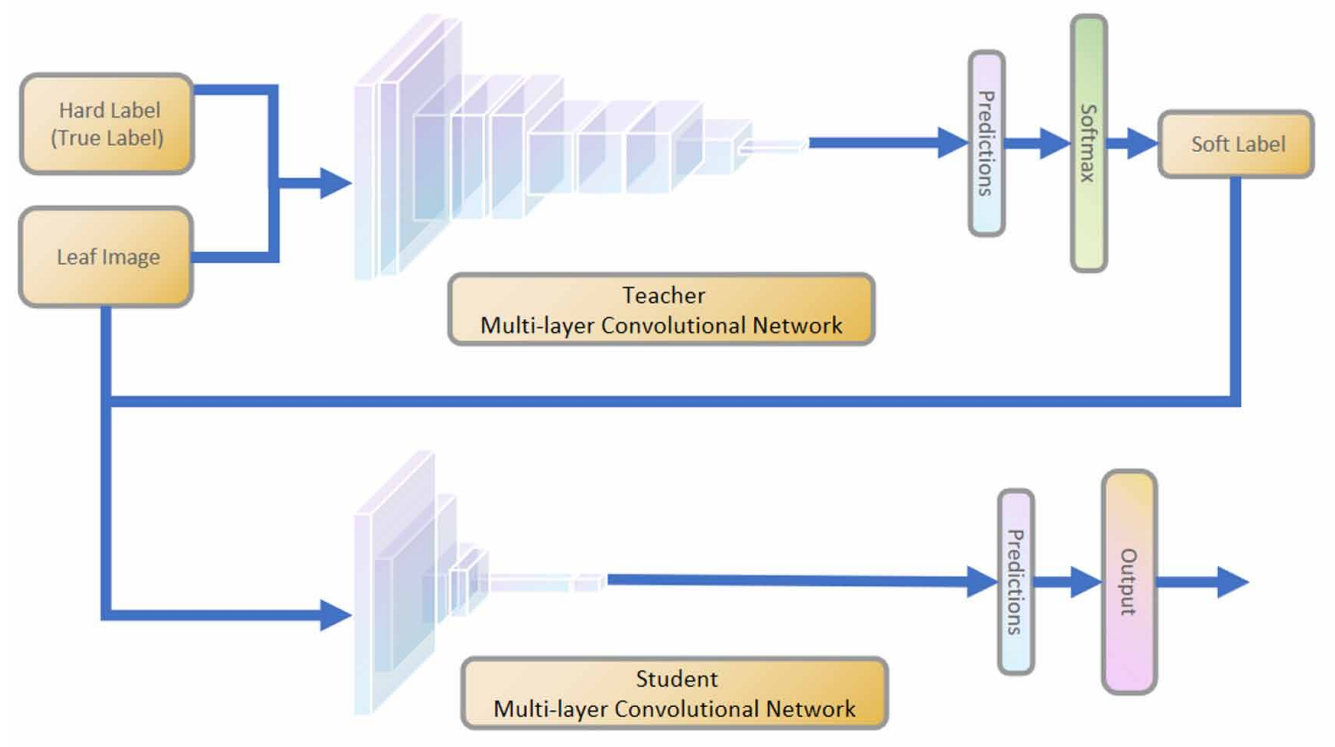

\section{Experiment Settings}

Alexnet, VGG16, GoogLeNet, and ResNet trained on ImageNet were used as pre-training models. The last layer was replaced, as stated above, to conduct transfer learning. The structure is shown in Figures 1a-1d. The models were renamed Fine-Alexnet, Fine-VGG16, Fine-GoogLeNet, and Fine-ResNet, respectively. After a high identification success rate was realized by the four new models, they were used as teacher network models in knowledge distillation to train each student network model. The structure of the student network models is shown in Figure 1(e). The output trained models were named Distillation-Alexnet, Distillation-VGG16, Distillation-GoogLeNet, and Distillation-ResNet. During the training process, one data set accuracy test was conducted for every ten model trainings. Moreover, for comparison, we randomly initialized the metrics parameter of Alexnet, and trained it to identify rare and endangered plant leaf images without using the pre-trained model. This is called CNN. At the same time, referring to methods described in the literature (Ibrahim et al., 2018), a rare and endangered plant leaf identification model based on an SVM was trained. To compare the lightweight models proposed in this article, we have trained leaf recognition models of rare and endangered plants based on the lightweight networks, MobileNet (Howard et al., 2017) and Xception (Chollet, 2017).The hardware configuration was CPU Intel 8700K, GPU NVIDA RTX2080Ti, and RAM 16G DDR4. Python was used as the programming language, and TensorFlow was the development framework.

The experiment was carried out in small batches, and each batch used 32 images for training/testing at the same time. The loss function optimizer used the Stochastic Gradient Descent algorithm, and the learning rate used a periodic decay update strategy. The initial learning rate was $1 \mathrm{e}-2$, the maximum learning rate was $1 \mathrm{e}-2$, the warm-up period was 3 epochs, the momentum was 0.9 , and the weight decay coefficient was $5 \mathrm{e}-6$.

\section{Comparison of Experiment Results}

The accuracy rate and loss variation curves for Fine-Alexnet, Fine-VGG16, Fine- GoogLeNet, and FineResNet in the image training and testing sets are shown in Figures 4-7. It is obvious that the accuracy 
rates in the training set and testing set were both stable when epoch was set to 120 . Loss rate was also stable when Epoch was 120. Fine-Alexnet realized an accuracy rate of $98.5 \%$ in the training set, and $97.1 \%$ in the testing set. Fine-VGG16 realized an accuracy rate of $98.2 \%$ in training and $96.5 \%$ in testing. The corresponding values for Fine-GoogLeNet were 99.2\% and 98.5\%, respectively; for Fine-ResNet, they were $99.5 \%$ and $94.7 \%$, respectively. The accuracy rates for Fine-VGG16 and Fine-ResNet were a bit lower than for Fine-Alexnet and Fine-GoogLeNet as the network depth of the former was much deeper than for the latter. In cases where there was an insufficient number of rare and endangered plant leaf images, Fine-VGG16 and Fine-ResNet had a higher risk of overfitting than did their counterparts.

The accuracy rate and loss variation curves for Distillation-Alexnet, Distillation-VGG16, DistillationGoogLeNet, and Distillation-ResNet in the rare and endangered plant leaf image training and testing sets are shown in Figures 8-11. Distillation-Alexnet showed a stable accuracy rate in both the training and testing sets when Epoch was 60. The loss rate was also stable when Epoch was 60. Meanwhile, the accuracy rates of Distillation-VGG16, Distillation-GoogLeNet, and Distillation-ResNet in the training and testing sets became stable when Epoch was 70. The loss rate also became stable when Epoch was 70. This was because the number of parameters for these three models was large, but the number was small for the student models. More iterations were required to learn knowledge of Fine-VGG16, FineGoogLeNet, and Fine-ResNet. The accuracy rates for Distillation-Alexnet were $97.1 \%$ in the training set and $96.3 \%$ in the testing set. The values for Distillation-VGG16 were $96.2 \%$ and $95.8 \%$, respectively. For Distillation-GoogLeNet and Distillation-ResNet, the respective accuracy rates were $95.1 \%$ and $92.7 \%$, and $89.7 \%$ and $90.1 \%$. The accuracy rates of the Distillation student models were very similar to those of the teacher models.

A comparison of the experiment results for the identification models is shown in Table 1. In the table, SVM was of the shortest training duration, but the lowest accuracy rate in the testing sets. This is because SVM has the smallest number of parameters, yet its feature extraction depends on artificial experience. The CNN method started training from the beginning. Therefore, it used the longest duration, yet its accuracy rate was disappointing. Fine-GoogLeNet had the highest accuracy rate in the testing set and a shorter duration than CNN. This reflects that transfer learning had not only saved the required training duration but also increased the accuracy rate for rare and endangered plant leaf identification. Fine-Alexnet and Fine-VGG16 were of similar accuracy to Fine-GoogLeNet. Fine-ResNet displayed the lowest accuracy rate in the testing set. Fine-ResNet had a longer training duration than the other fine-tuning networks, likely because Fine-ResNet involved a larger number of parameters. Distillation-Alexnet, Distillation-VGG16, and Distillation-GoogLeNet presented accuracy rates similar to Fine-Alexnet, Fine-VGG16, and Fine-GoogLeNet after knowledge distillation, which indicates that knowledge about rare and endangered plant leaves had already been transferred to the lightweight model. The accuracy of Distillation-ResNet is not ideal, which is related to the lack of residual modules in the student model. The lightweight networks, MobileNet and Xception, achieved better accuracy than the distillation student model, but the training time was longer than the distillation student model.

A comparison of the complexity of the teacher and student models is shown in Table 2. In the table, floating point operations per second (FLOPs) is generally understood as calculating the number of network models and is used to measure model complexity. We used the keras-FLOPS library to calculate the FLOPs of the teacher and student models. The total number of parameters of DistillationAlexnet was only $19.00 \%$ of those for Fine-Alexnet. Its FLOPs was $32.70 \%$ of Fine-Alexnet. The total number of parameters of Distillation-VGG16 was 5.64\% of Fine-VGG16, and its FLOPs was 2.08\% of Fine-VGG16. For Distillation-GoogLeNet, the respective values were 5.70\% and $12.83 \%$ of FineGoogLeNet, and for Distillation-ResNet, they were 5.30\% and 7.30\% of Fine-ResNet. For the distillation student model, the values were $6.21 \%$ and $7.15 \%$ of Xception, and the distillation student model was $36.23 \%$ and $58.90 \%$, respectively, of MobileNet. This indicates that the lightweight rare and endangered plant leaf model proposed in this paper balanced identification accuracy rate and computing resource utilization rate, and realized a balance between parameter numbers and identification accuracy rate. 
International Journal of Agricultural and Environmental Information Systems Volume 12 - Issue 4

Figure 3. Some data in the experiment data set of rare and endangered plant leaves

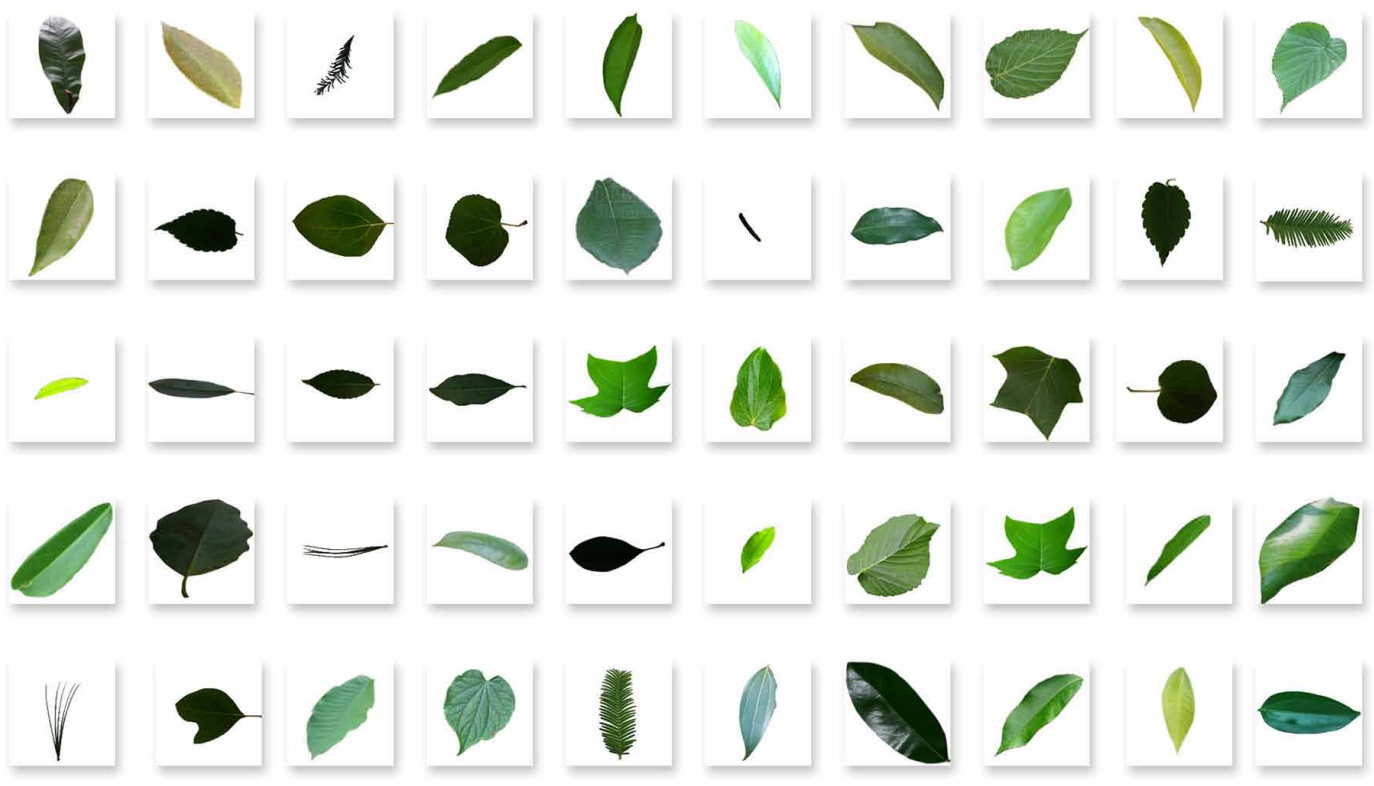

Figure 4a. Experiment results for fine-alexnet

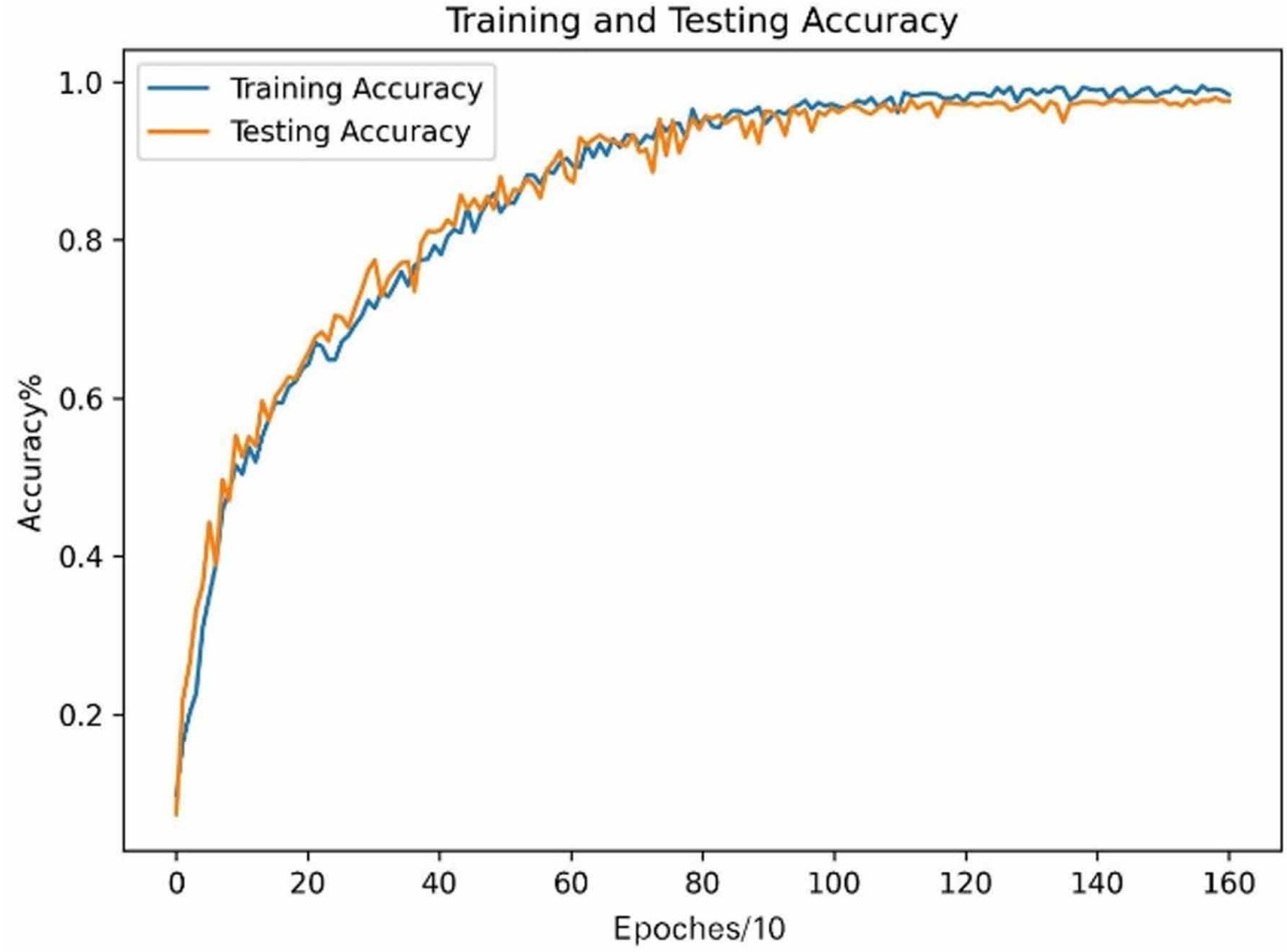




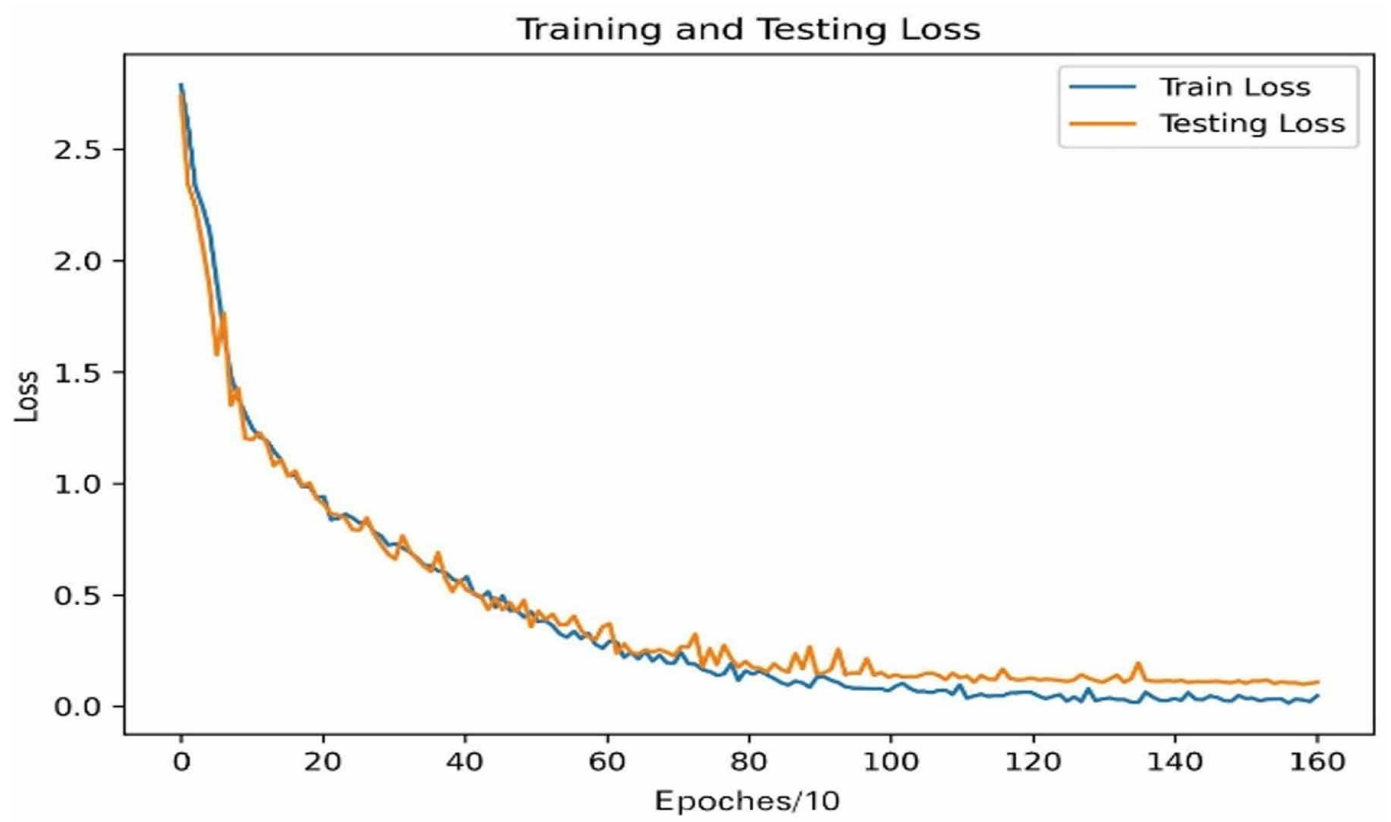

Figure 5a. Experiment results for Fine-VGG16

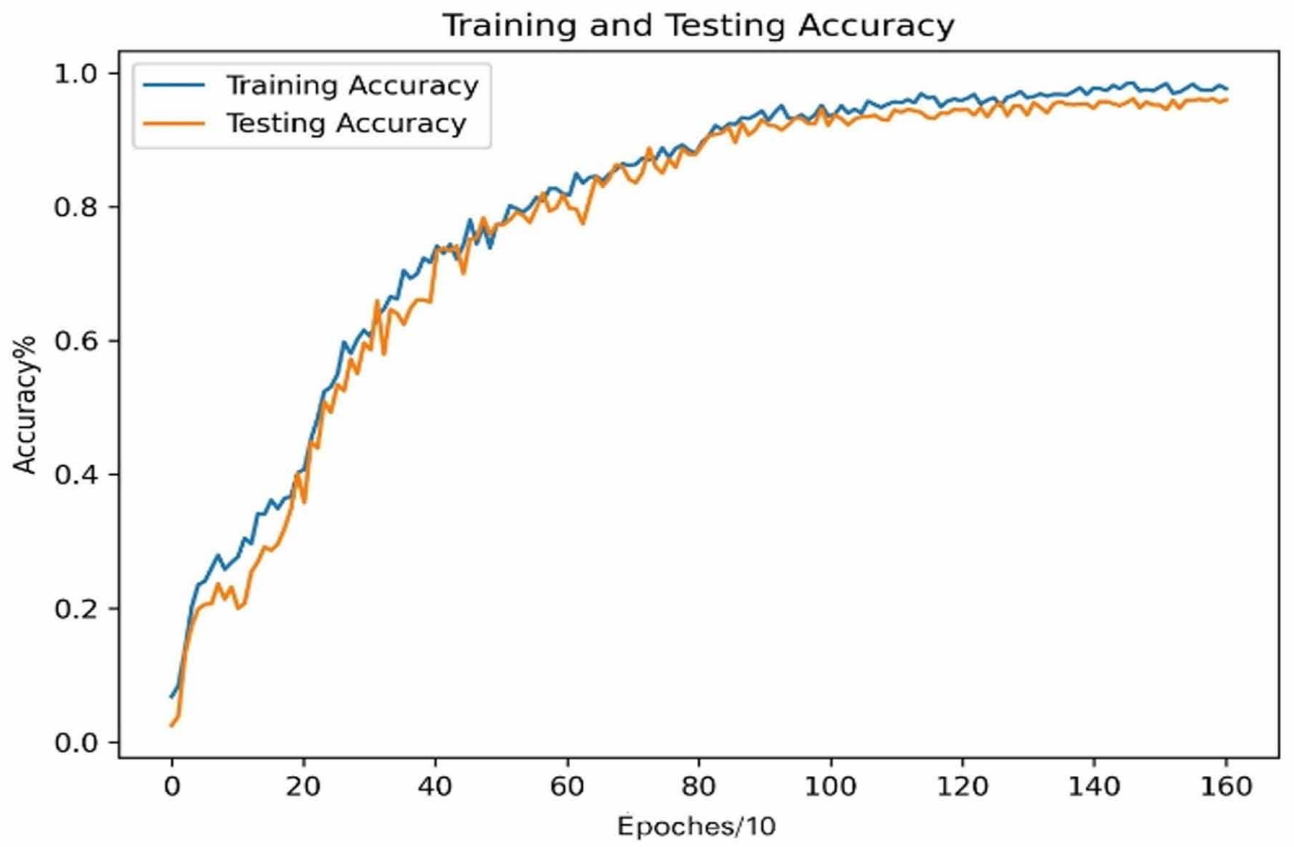


International Journal of Agricultural and Environmental Information Systems

Volume 12 Issue 4

Figure 5b. Experiment results for Fine-VGG16

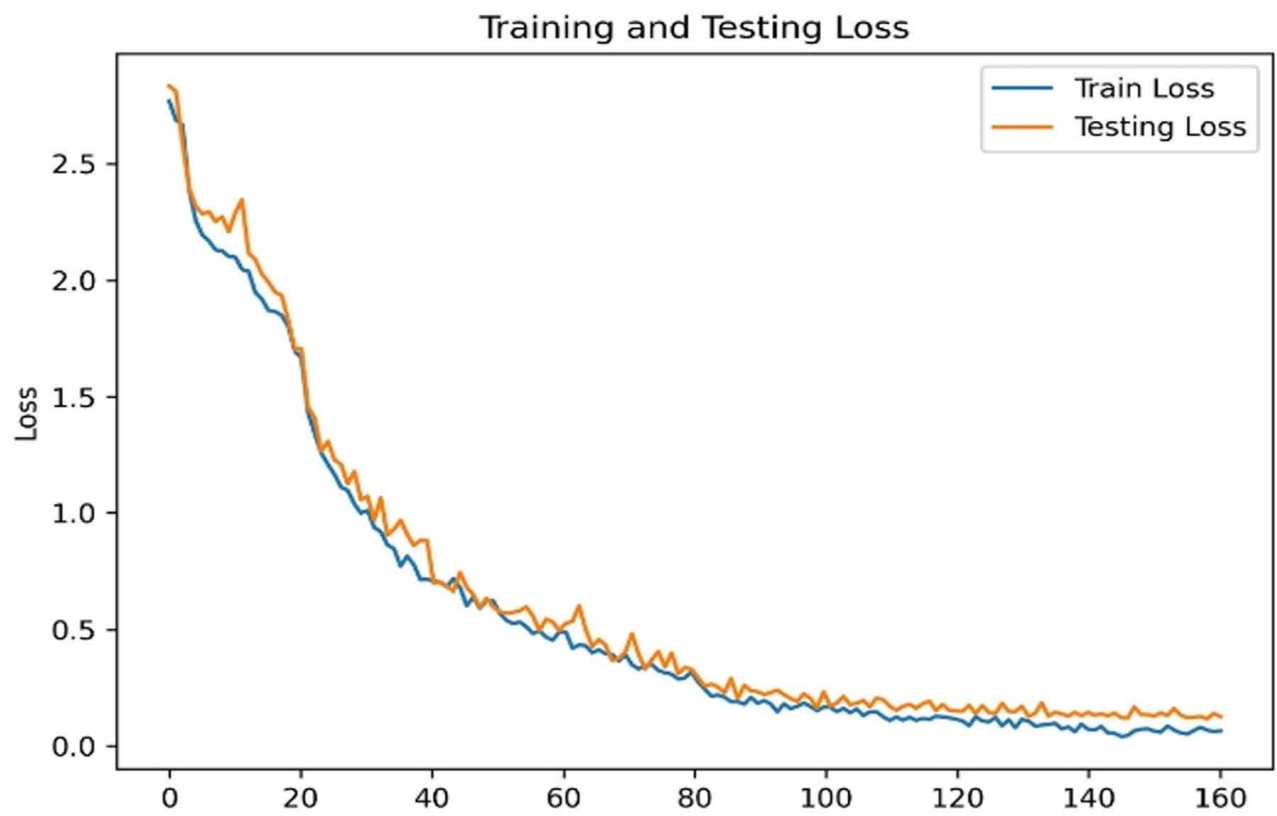

Figure 6a. Experiment results for Fine-GoogLeNet

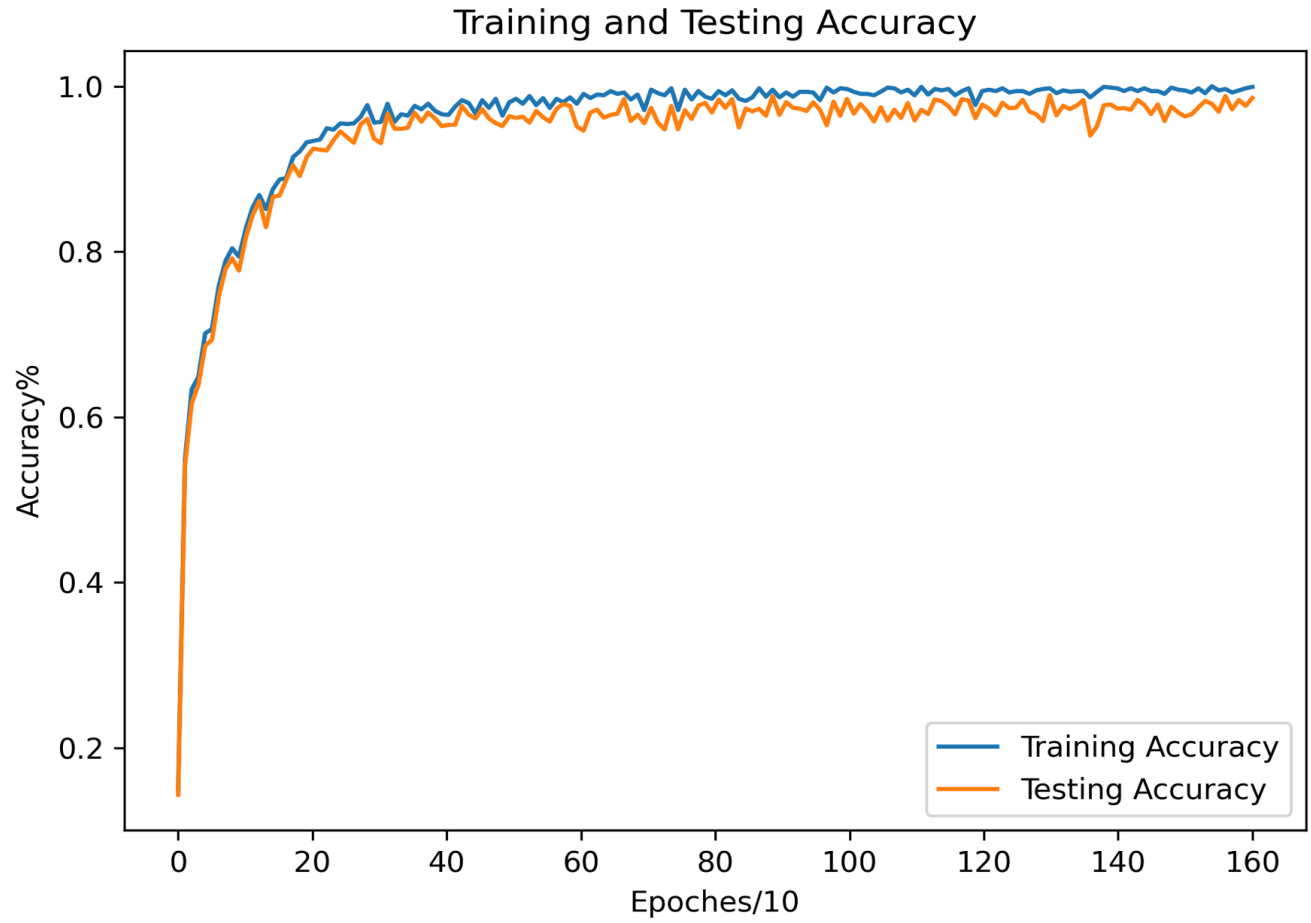


Figure 6b. Experiment results for Fine-GoogLeNet

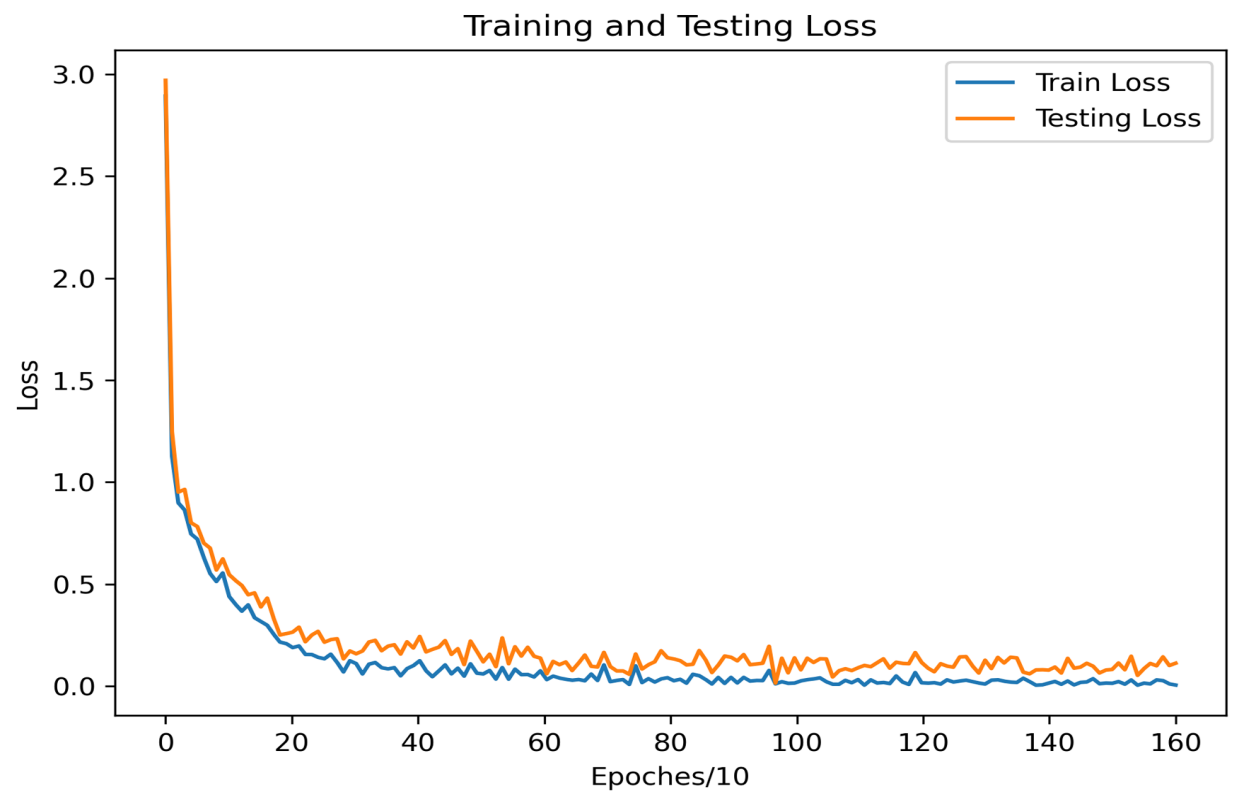

Figure 7a. Experiment results for fine-resnet

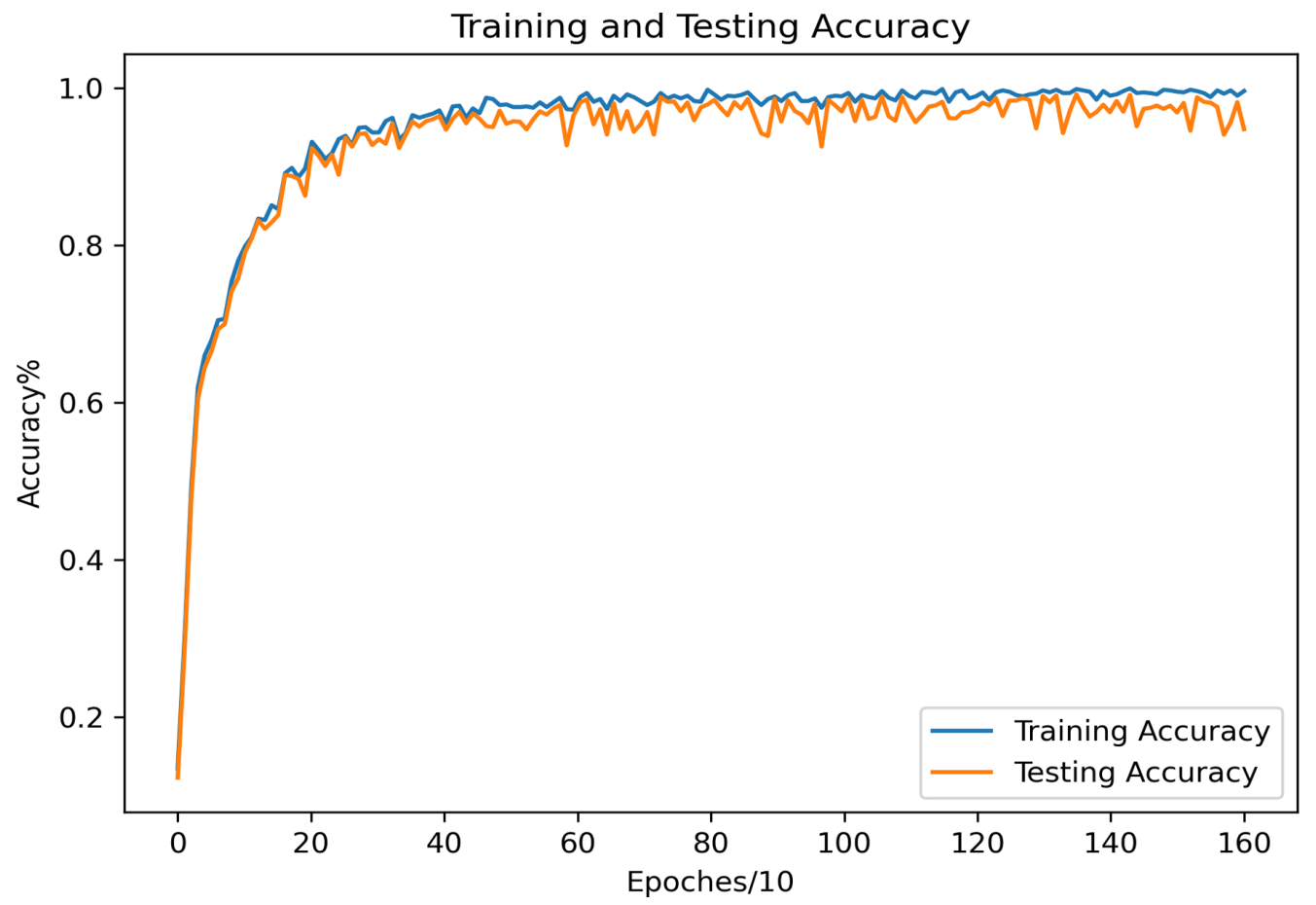


International Journal of Agricultural and Environmental Information Systems

Volume 12 Issue 4

Figure 7b. Experiment results for fine-resnet

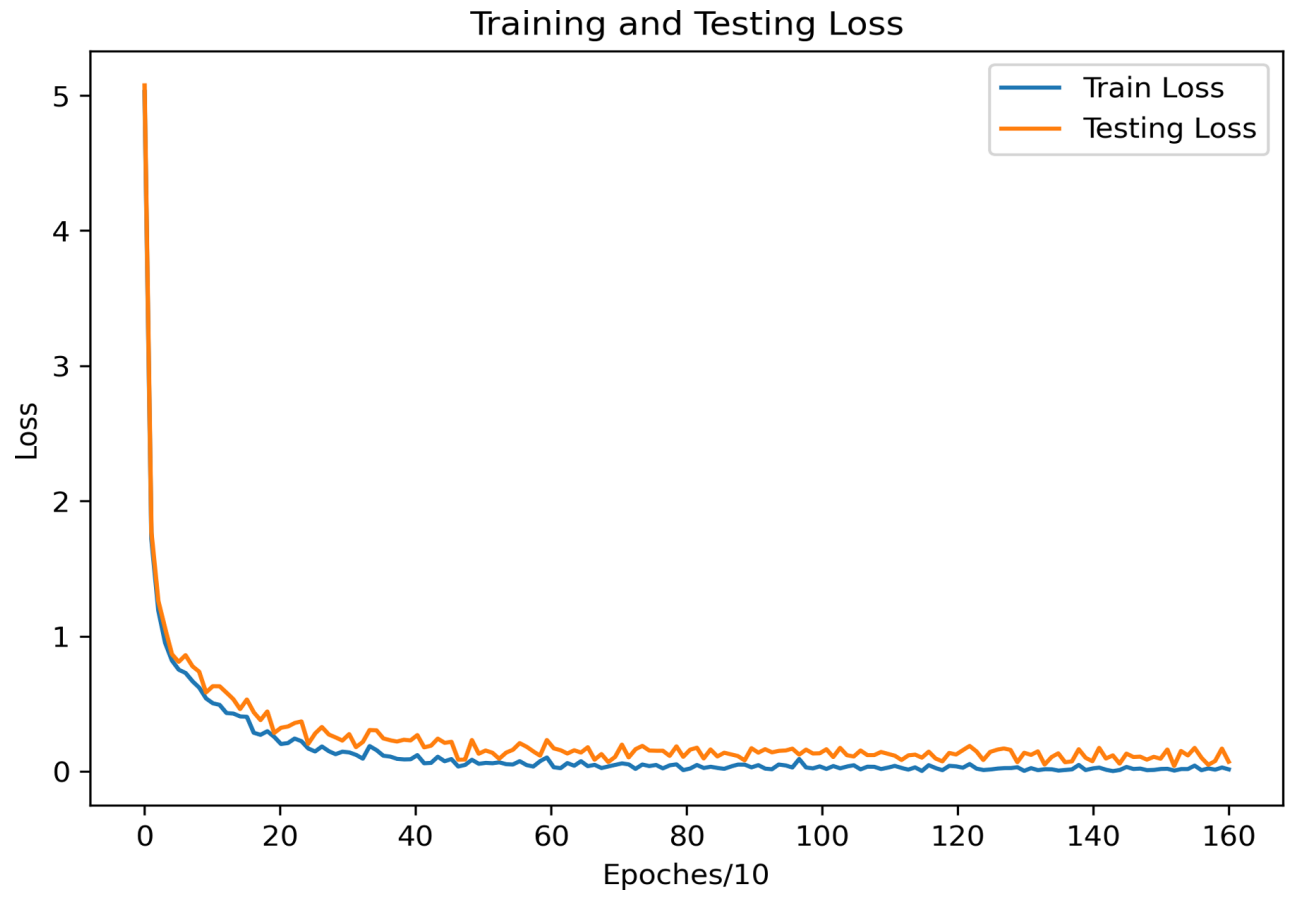

Figure 8a. Experiment results for Distillation-Alexnet

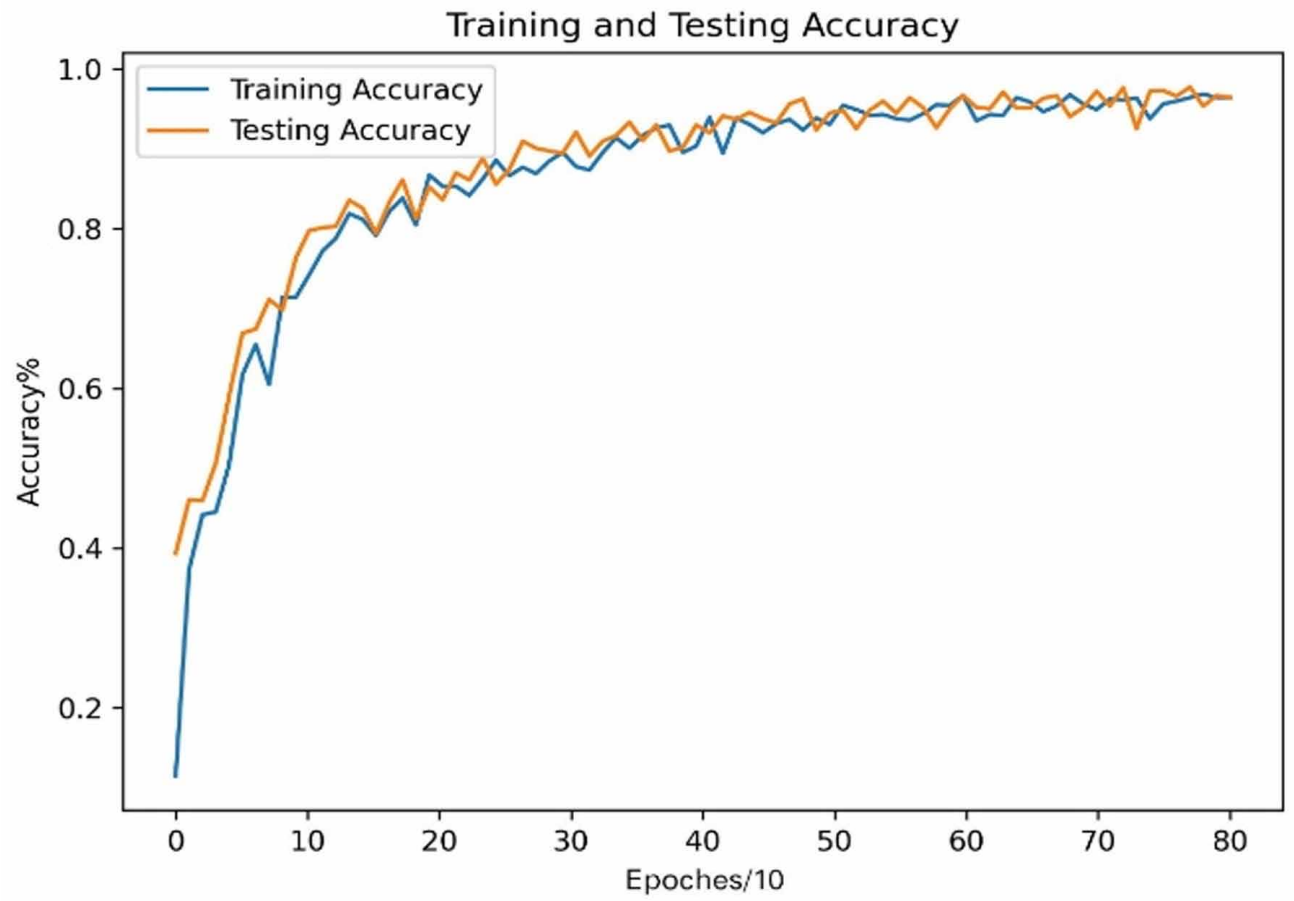


Figure 8b. Experiment results for Distillation-Alexnet

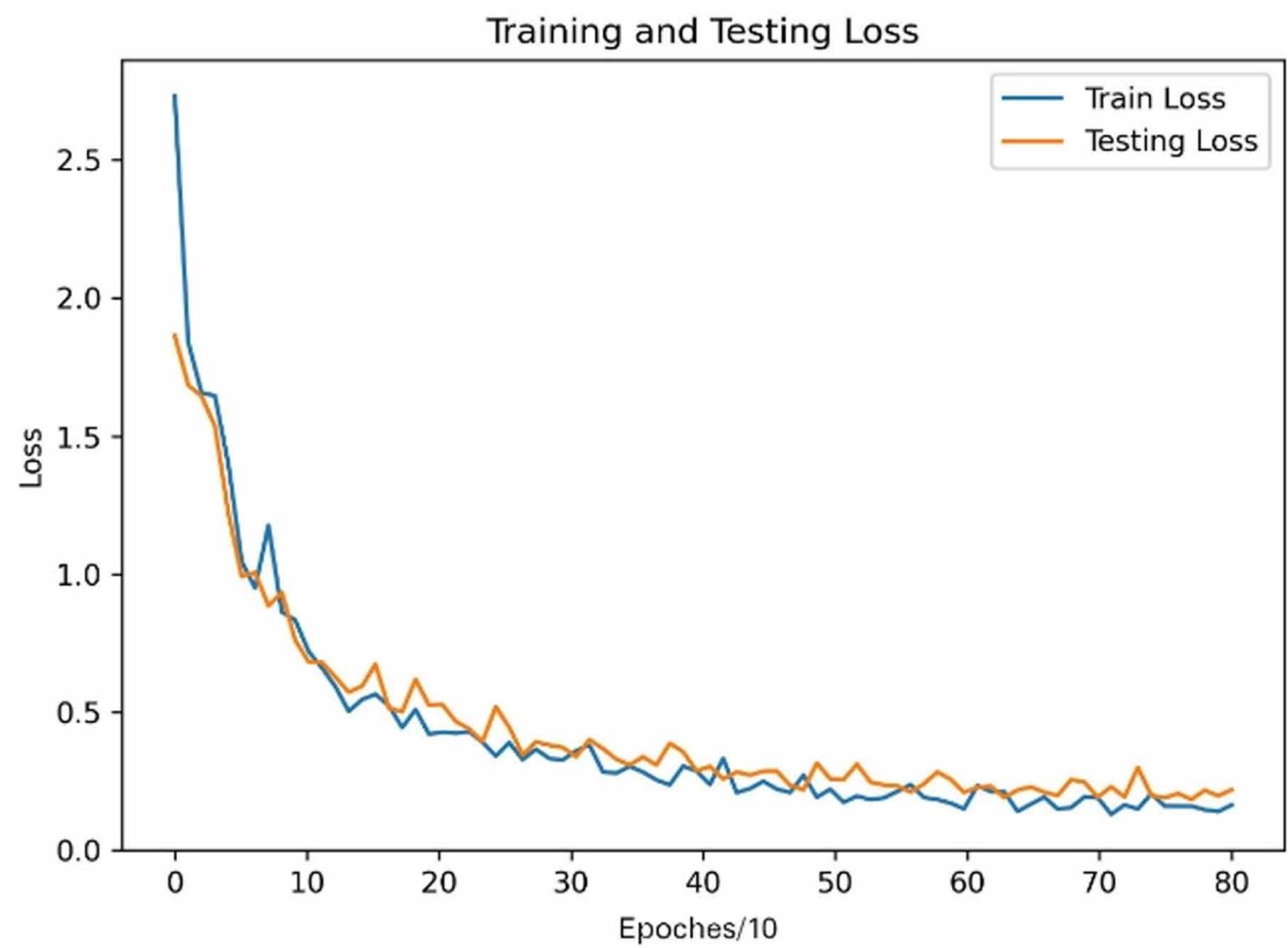

Figure 9a. Experiment results for Distillation-VGG16

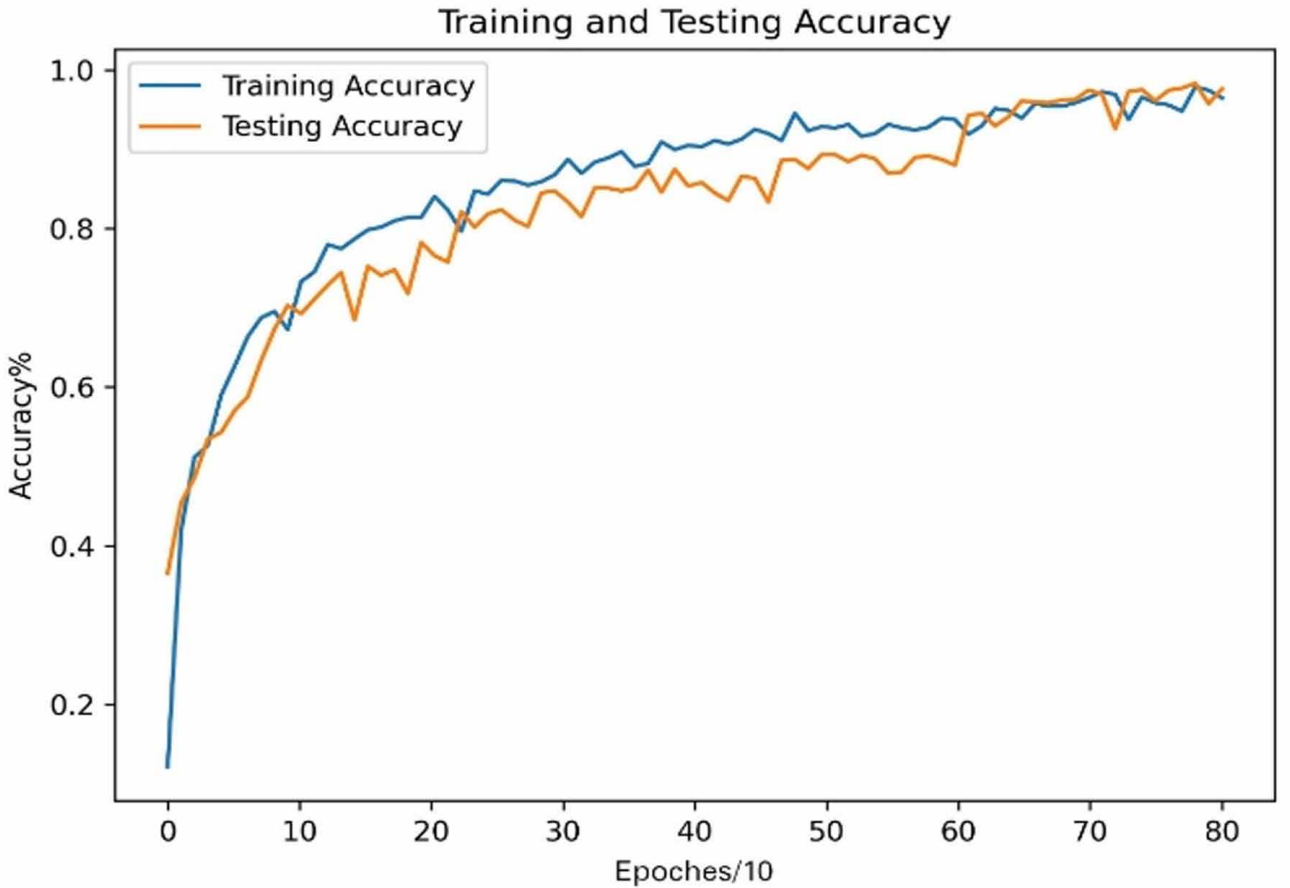


International Journal of Agricultural and Environmental Information Systems

Volume 12 Issue 4

Figure 9b. Experiment results for Distillation-VGG16

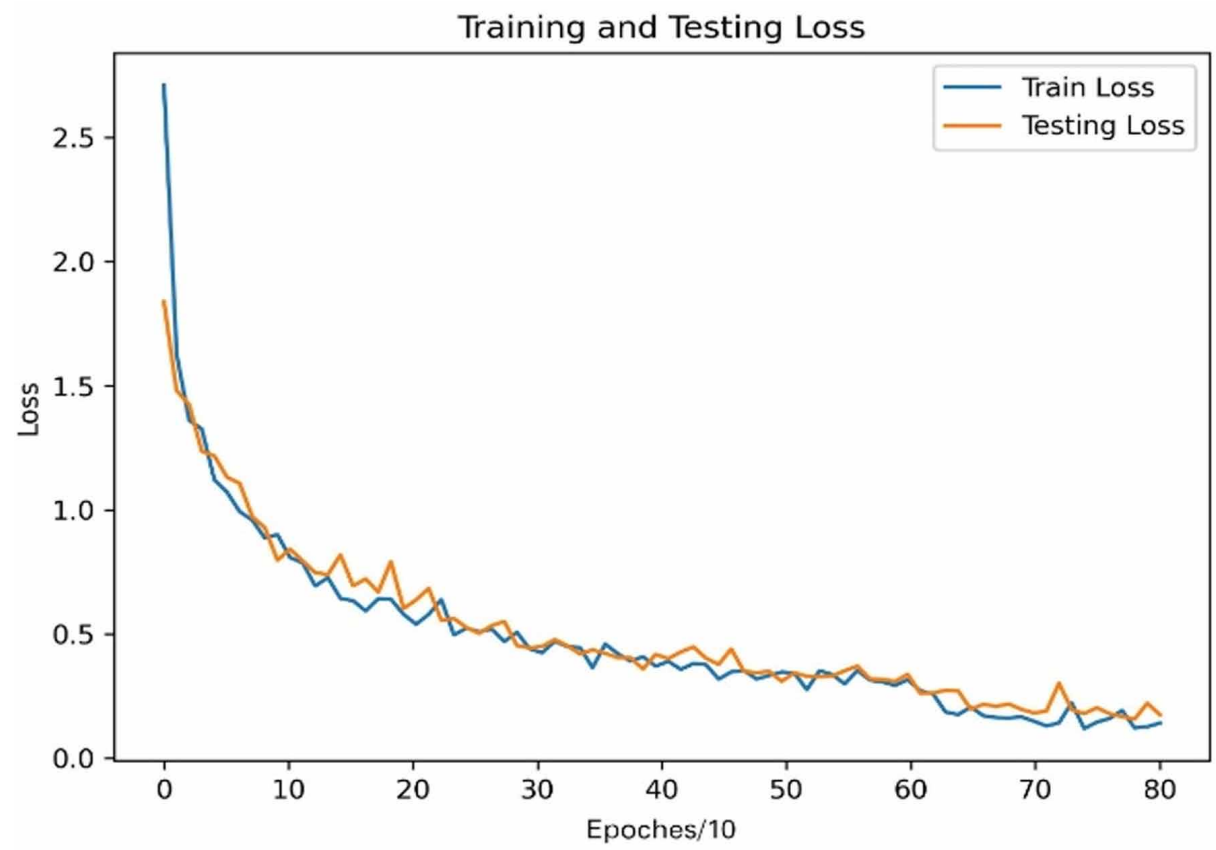

Figure 10a. Experiment results for Distillation-GoogLeNet

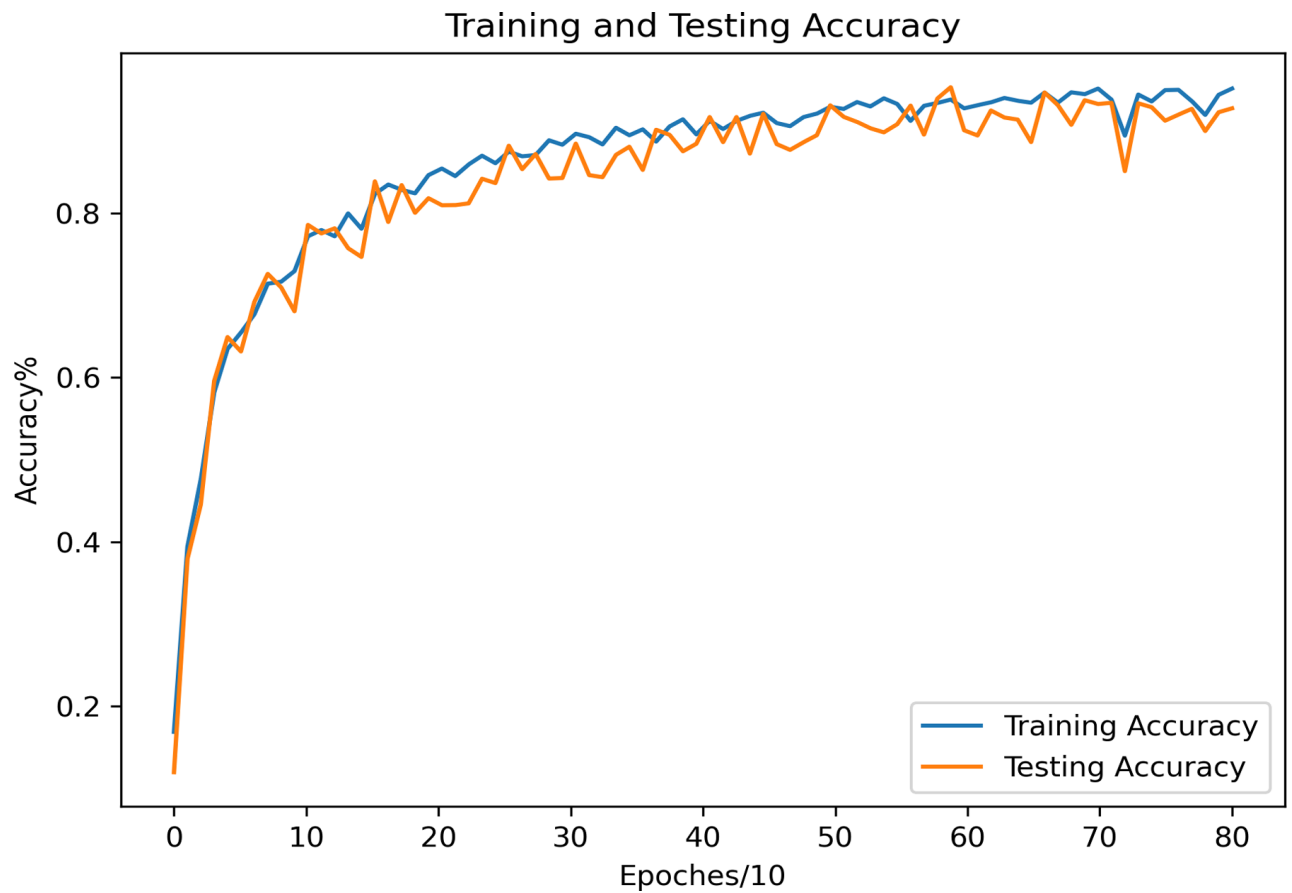


Figure 10b. Experiment results for Distillation-GoogLeNet

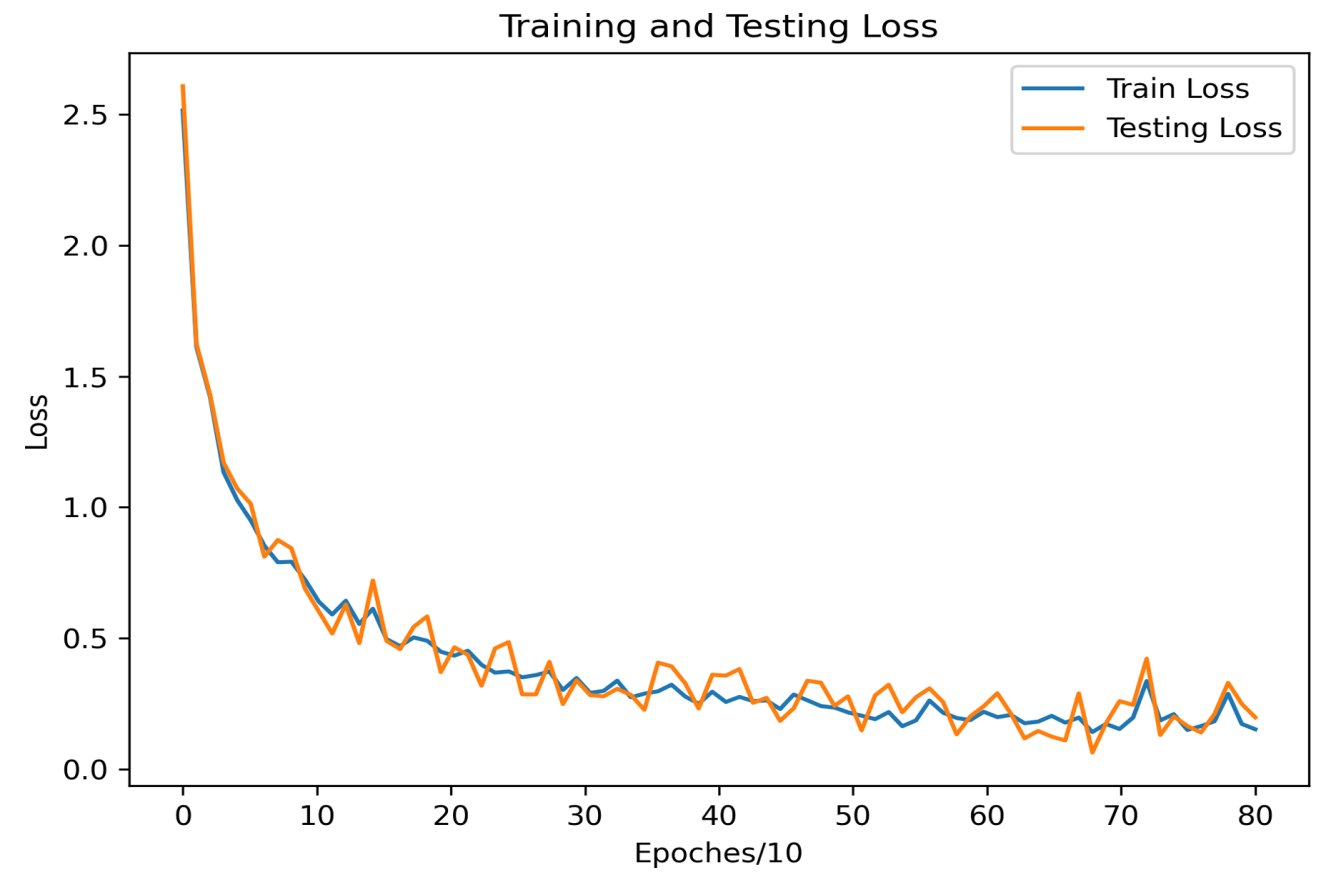

Figure 11a. Experiment results for distillation-resnet

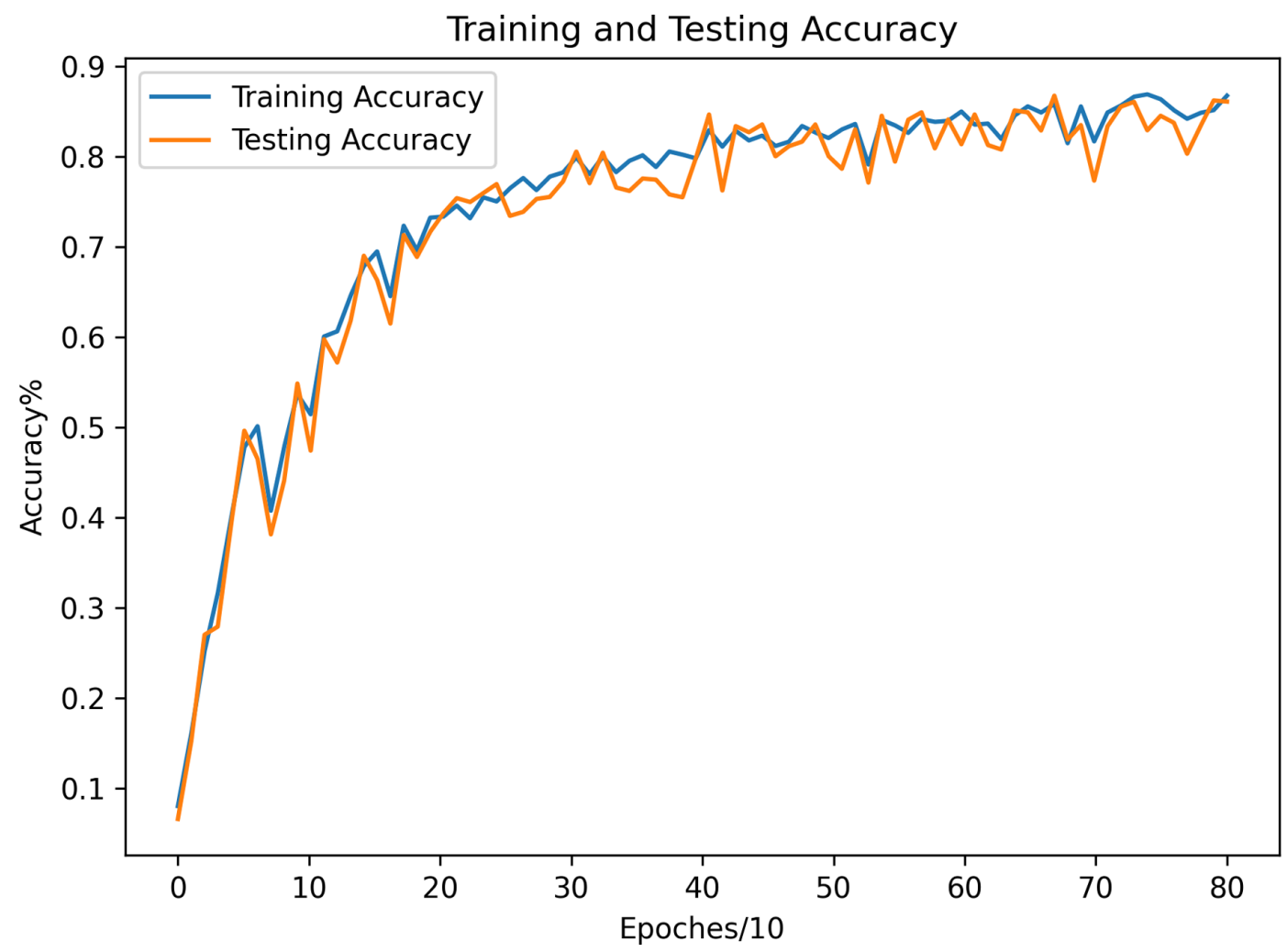


Training and Testing Loss

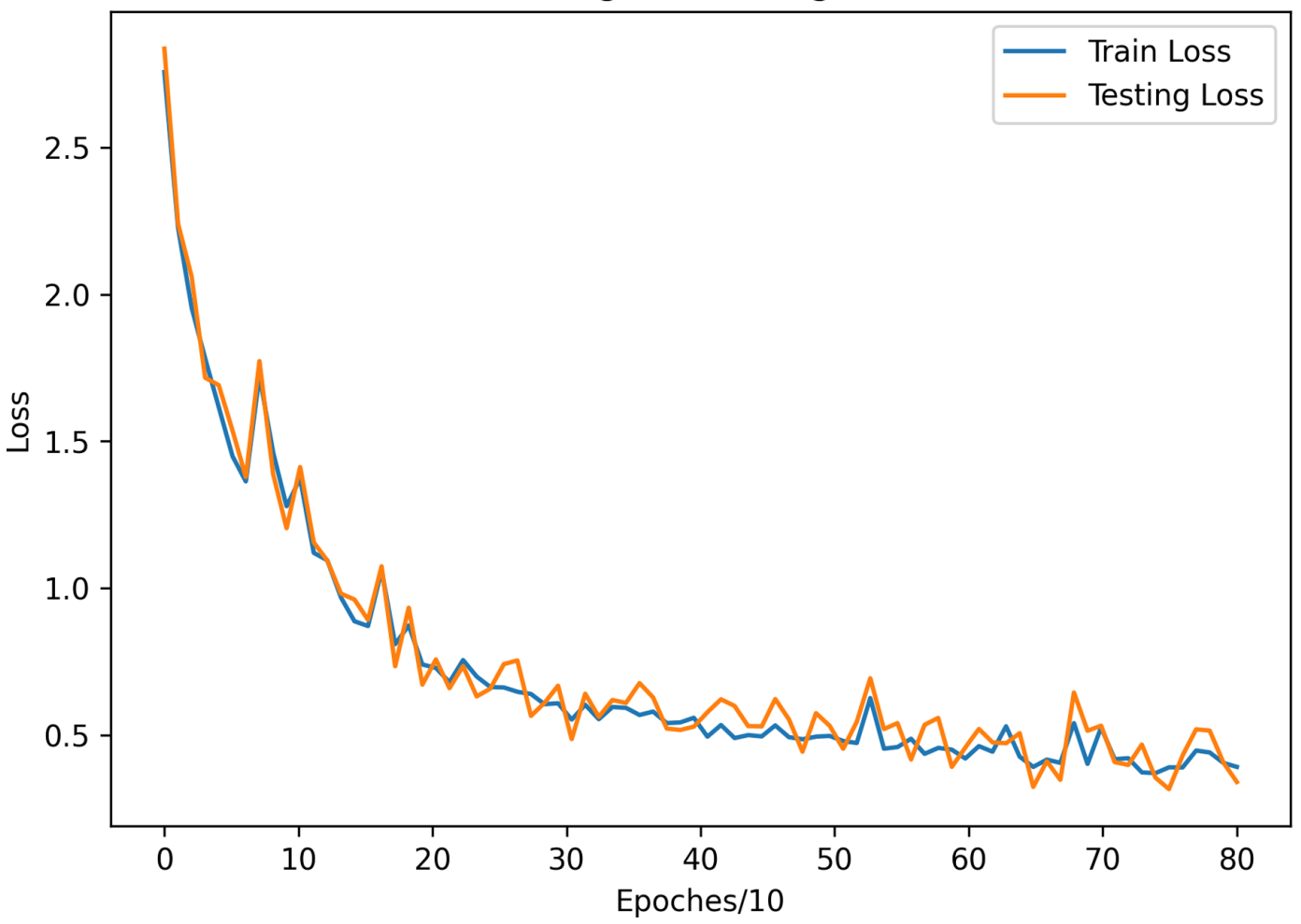

Table 1. Comparison of experiment results for identification models

\begin{tabular}{|l|l|l|l|}
\hline Methodology & $\begin{array}{l}\text { Training duration } \\
\text { (hours) }\end{array}$ & $\begin{array}{l}\text { Accuracy rate in training } \\
\text { set (\%) }\end{array}$ & $\begin{array}{l}\text { Accuracy rate in testing } \\
\text { set (\%) }\end{array}$ \\
\hline SVM & 0.5 & 0.84 & 0.81 \\
\hline CNN & 3.1 & 0.95 & 0.92 \\
\hline Fine-Alexnet & 1.9 & 0.985 & 0.971 \\
\hline Fine-VGG16 & 2.4 & 0.982 & 0.965 \\
\hline Fine-GoogLeNet & 1.8 & 0.992 & 0.985 \\
\hline Fine-ResNet & 2.5 & 0.995 & 0.947 \\
\hline MobileNet & 1.4 & 0.987 & 0.969 \\
\hline Xception & 3.6 & 0.981 & 0.967 \\
\hline Distillation-Alexnet & 0.5 & 0.971 & 0.963 \\
\hline Distillation-VGG16 & 0.6 & 0.962 & 0.958 \\
\hline Distillation-GoogLeNet & 0.7 & 0.951 & 0.927 \\
\hline Distillation-ResNet & 1.0 & 0.897 & 0.901 \\
\hline
\end{tabular}


Table 2. Comparison of the complexity of the teacher and student models

\begin{tabular}{|l|l|l|l|}
\hline Methodology & Total parameter amount & Layer amount & FLOPs \\
\hline Fine-Alexnet & $7,173,520$ & 13 & 0.844 \\
\hline Fine-VGG16 & $24,170,320$ & 23 & 13.260 \\
\hline Fine-GoogLeNet & $23,917,360$ & 314 & 2.150 \\
\hline Fine-ResNet & $25,702,288$ & 178 & 3.780 \\
\hline MobileNet & $3,761,872$ & 90 & 0.468 \\
\hline Xception & $21,918,776$ & 135 & 3.860 \\
\hline Distillation student model & $1,363,088$ & 8 & 0.276 \\
\hline
\end{tabular}

\section{CONCLUSION}

This paper proposed a rare and endangered plant leaf identification method based on transfer learning and knowledge distillation. It, first, used the transfer learning method, which applied Alexnet, VGG16, GoogLeNet, and ResNet as pre-trained models, and replaced the last layer to enable transfer learning. Then, it used knowledge distillation to transfer knowledge from Alexnet, VGG16, GoogLeNet, and ResNet into the lightweight network model. Finally, the experiments proved that the lightweight model proposed in this paper can achieve precise recognition accuracy while using simple calculations of resources. The method presented here has not only realized high accuracy in rare and endangered plant leaf identification, but also reduced the complexity of these identification models. It offers dependable technology support for the operation of identification models in mobile smart terminals and embedded devices. In the future, we plan to continue expanding the data set to test and improve the current algorithm, further promoting the generalizability of the identification model in rare and endangered plant leaf identification.

\section{ACKNOWLEDGMENT}

This research was supported by Population Health Informatization at the Hebei Province Engineering Technology Research Center, School-level scientific research project of Hebei North University (grant number YB2020002), and S\&T Program of Hebei (grant number 216Z0302G). Lin Wu and Jingjing Yang contributed equally to this work. Xiao Zhang is the corresponding author of this article. 


\section{REFERENCES}

Beikmohammadi, A., \& Faez, K. (2018). Leaf classification for plant recognition with deep transfer learning. Paper presented at the 2018 4th Iranian Conference on Signal Processing and Intelligent Systems (ICSPIS). doi:10.1109/ICSPIS.2018.8700547

Ceballos, G., Ehrlich, P. R., \& Raven, P. H. (2020). Vertebrates on the brink as indicators of biological annihilation and the sixth mass extinction. Proceedings of the National Academy of Sciences of the United States of America, 117(24), 13596-13602. doi:10.1073/pnas.1922686117 PMID:32482862

Chaki, J., Dey, N., Moraru, L., \& Shi, F. (2019). Fragmented plant leaf recognition: Bag-of-features, fuzzycolor and edge-texture histogram descriptors with multi-layer perceptron. Optik (Stuttgart), 181, 639-650. doi:10.1016/j.ijleo.2018.12.107

Chollet, F. (2017). Xception: Deep learning with depthwise separable convolutions. Proceedings of the IEEE conference on computer vision and pattern recognition. doi:10.1109/CVPR.2017.195

Fan, D., Huang, J., Hu, H., Sun, Z., Cheng, S., Kou, Y., \& Zhang, Z. (2018). Evolutionary Hotspots of Seed Plants in Subtropical China: A Comparison With Species Diversity Hotspots of Woody Seed Plants. Frontiers in Genetics, 9(333), 333. Advance online publication. doi:10.3389/fgene.2018.00333 PMID:30177954

Gogul, I., \& Kumar, V. S. (2017). Flower species recognition system using convolution neural networks and transfer learning. Paper presented at the 2017 Fourth International Conference on Signal Processing, Communication and Networking (ICSCN). doi:10.1109/ICSCN.2017.8085675

He, K., Zhang, X., Ren, S., \& Sun, J. (2016). Deep Residual Learning for Image Recognition. Paper presented at the computer vision and pattern recognition. doi:10.1109/CVPR.2016.90

Hewitt, C., \& Mahmoud, M. (2018). Shape-only Features for Plant Leaf Identification. arXiv:1811.08398. Retrieved from https://ui.adsabs.harvard.edu/abs/2018arXiv181108398H

Hinton, G., Vinyals, O., \& Dean, J. (2015). Distilling the Knowledge in a Neural Network. arXiv:1503.02531. Retrieved from https://ui.adsabs.harvard.edu/abs/2015arXiv150302531H

Howard, A.G., Zhu, M., Chen, B., Kalenichenko, D., Wang, W., Weyand, T., Andreetto, M., \& Adam, H. (2017). MobileNets: Efficient Convolutional Neural Networks for Mobile Vision Applications. ArXiv, abs/1704.04861.

Ibrahim, Z., Sabri, N., \& Abu Mangshor, N. N. (2018). Leaf Recognition using Texture Features for Herbal Plant Identification. Indonesian Journal of Electrical Engineering and Computer Science, 9(1), $152-156$. doi:10.11591/ijeecs.v9.i1.pp152-156

Islama, M., Yousuf, S., \& Billah, M. (2019). Automatic plant detection using HOG and LBP features with SVM. Int. J. Comput, 33(1), 26-38.

Kadir, A. (2014). A Model of Plant Identification System Using GLCM, Lacunarity And Shen Features. Research Journal of Pharmaceutical, Biological and Chemical Sciences, 5(2), 1-10.

Kadir, A., Nugroho, L. E., Susanto, A., \& Santosa, P. I. (2011). Leaf Classification Using Shape, Color, and Texture Features. International Journal of Computer Trends and Technology, 1(3), 225-230.

Kadir, A., Nugroho, L. E., Susanto, A., \& Santosa, P. I. (2013). Neural Network Application on Foliage Plant Identification. Computerence, 11(8-9), 15-22.

Kaya, A., Keceli, A. S., Catal, C., Yalic, H. Y., Temucin, H., \& Tekinerdogan, B. (2019). Analysis of transfer learning for deep neural network based plant classification models. Computers and Electronics in Agriculture, 158, 20-29. doi:10.1016/j.compag.2019.01.041

Krizhevsky, A., Sutskever, I., \& Hinton, G. E. (2017). ImageNet classification with deep convolutional neural networks. Commun. ACM, 60(6), 84-90. 10.1145/3065386

Kumar, N. et al.. (2012). Leafsnap: A Computer Vision System for Automatic Plant Species Identification. In A. Fitzgibbon, S. Lazebnik, P. Perona, Y. Sato, \& C. Schmid (Eds.), Lecture Notes in Computer Science: Vol. 7573. Computer Vision - ECCV 2012. ECCV 2012. Springer., https://doi.org/10.1007/978-3-642-33709-3_36. 
Lee, S. H., Chan, C. S., Mayo, S. J., \& Remagnino, P. (2017). How deep learning extracts and learns leaf features for plant classification. Pattern Recognition, 71, 1-13. doi:10.1016/j.patcog.2017.05.015

Li, B., Li, M., Li, J., Fan, P., Ni, Q., Lu, J., Zhou, X., Long, Y., Jiang, Z., Zhang, P., Huang, Z., Huang, C., Jiang, X., Pan, R., Gouveia, S. F., Dobrovolski, R., Grueter, C., Oxnard, C., Groves, C., \& Garber, P. et al. (2018). The primate extinction crisis in China: Immediate challenges and a way forward. Biodiversity and Conservation, $27,3301-3327$.

Lin, C., Ding, Q., Tu, W., Huang, J., \& Liu, J. (2019). Fourier Dense Network to Conduct Plant Classification Using UAV-Based Optical Images. IEEE Access: Practical Innovations, Open Solutions, 7, 17736-17749. doi:10.1109/ACCESS.2019.2895243

Mata-Montero, E., \& Carranza-Rojas, J. (2015). A texture and curvature bimodal leaf recognition model for identification of Costa Rican plant species, 2015 Latin American Computing Conference (CLEI), pp. 1-12, doi: doi:10.1109/CLEI.2015.7360026

Mounce, R., Smith, P., \& Brockington, S. (2017). Ex situ conservation of plant diversity in the world's botanic gardens. Nature Plants, 3, 795-802. doi:10.1038/s41477-017-0019-3

Narzullaev, O. K. (2021). Protection Of Biological Resources And Problems Of Legal Regulation Of The Use Of Biodiversity. The American Journal of Political Science Law and Criminology, 3(2), 1-6. doi:10.37547/ tajpslc/Volume03Issue02-01

Parvatikar, S., \& Parasar, D. (2021). Categorization of Plant Leaf Using CNN. In V. E. Balas, V. B. Semwal, A. Khandare, \& M. Patil (Eds.), Intelligent Computing and Networking. Lecture Notes in Networks and Systems (Vol. 146). Springer., https://doi.org/10.1007/978-981-15-7421-4_7.

Pawara, P., Okafor, E., Surinta, O., Schomaker, L., \& Wiering, M. (2017). Comparing Local Descriptors and Bags of Visual Words to Deep Convolutional Neural Networks for Plant Recognition. In Proceedings of the 6th International Conference on Pattern Recognition Applications and Methods - ICPRAM, ISBN 978-989-758222-6; ISSN 2184-4313, pages 479-486. DOI: 10.5220/0006196204790486

Qassim, H., Verma, A., \& Feinzimer, D. (2018). Compressed residual-VGG16 CNN model for big data places image recognition. 2018 IEEE 8th Annual Computing and Communication Workshop and Conference (CCWC), 169-175.

Roberts, L., Hassan, A., Elamer, A., \& Nandy, M. (2021). Biodiversity and extinction accounting for sustainable development: A systematic literature review and future research directions. Business Strategy and the Environment, 30(1), 705-720. doi:10.1002/bse.2649

Saleem, G., Akhtar, M., Ahmed, N., \& Qureshi, W. S. (2019). Automated analysis of visual leaf shape features for plant classification. Computers and Electronics in Agriculture, 157, 270-280. doi:10.1016/j.compag.2018.12.038

Singh, N. P., Nagahma, T., Yadav, P., \& Yadav, D. (2018). Feature based leaf identification. Paper presented at the 2018 5th IEEE Uttar Pradesh section international conference on electrical, electronics and computer engineering (UPCON). doi:10.1109/UPCON.2018.8596921

Szegedy, C., Vanhoucke, V., Ioffe, S., Shlens, J., \& Wojna, Z. (2016). Rethinking the inception architecture for computer vision. Proceedings of the IEEE conference on computer vision and pattern recognition. doi:10.1109/ CVPR.2016.308

Xu, Y., Shen, Z., Ying, L., Wang, Z., Huang, J., Zang, R., \& Jiang, Y. (2017). Hotspot analyses indicate significant conservation gaps for evergreen broadleaved woody plants in China. Scientific Reports, 7(1), 1859. 10.1038/ s41598-017-02098-0

Yang, C. (2021). Plant leaf recognition by integrating shape and texture features. Pattern Recognition, 112, 107809. doi:10.1016/j.patcog.2020.107809

Yao, Z., Guo, J., Jin, C., \& Liu, Y. (2021). Endangered mechanisms for the first-class protected Wild Plants with Extremely Small Populations in China [J]. Biodiv Sci, 29(3), 394-408.

Zhang, Q., Zeng, S., \& Zhang, B. (2019). Initial investigation of different classifiers for plant leaf classification using multiple features. Paper presented at the Eleventh International Conference on Digital Image Processing (ICDIP 2019). doi:10.1117/12.2539654 
Lin Wu was born in Hebei. She is currently pursuing a master's degree in agricultural engineering and information technology from the School of Science and Engineering, Hebei North University. Her research interests include agricultural informatics and machine learning.

Jingjing Yang is currently an Associate Professor with the School of Information Science and Engineering, Hebei North University. He is currently pursuing a Ph.D. degree at Chengdu Institute of Computer Applications, Chinese Academy of Sciences. His research focuses on machine learning and privacy protection. He applies these techniques to a wide range of real-world problems, for both academic research and industrial application.

Zhihao Gu was born in Hebei, China. He is currently pursuing a B.S. degree from the School of Science and Engineering, Hebei North University. His research interests include machine learning and agricultural informatics.

Jiaqian Guo was born in Hebei, China. She is currently pursuing a B.S. degree from the School of Information Science and Engineering, Hebei North University. Her research interests include machine learning and deep learning. She has participated in one provincial scientific research project.

Xiao Zhang is currently a Professor at Hebei North University. He has published three books and more than 30 refereed articles and has provided consulting to many major companies worldwide. For a long time, he has been engaged in the research and development of efficient and highly reliable computing and reasoning theories and tools. His research fields involve machine learning, agricultural informatics, medical informatics, and their intersection, fusion, and application. 